\title{
YVES GUIVARC'H
}

\section{Compactifications of Symmetric Spaces and Positive} Eigenfunctions of the Laplacian

Publications de l'Institut de recherche mathématiques de Rennes, 1994, fascicule 2 «Fascicule de probabilités », , p. 1-63

<http://www.numdam.org/item?id=PSMIR_1994 2_A2_0>

(C) Département de mathématiques et informatique, université de Rennes, 1994, tous droits réservés.

L'accès aux archives de la série « Publications mathématiques et informatiques de Rennes » implique l'accord avec les conditions générales d'utilisation (http://www.numdam.org/conditions). Toute utilisation commerciale ou impression systématique est constitutive d'une infraction pénale. Toute copie ou impression de ce fichier doit contenir la présente mention de copyright.

\section{NumDAm}

Article numérisé dans le cadre du programme Numérisation de documents anciens mathématiques http://www.numdam.org/ 


\title{
COMPACTIFICATIONS OF SYMIMETRIC SPACES
}

\section{AND POSITIVE EIGENFUNCTIONS OF THE LAPLACIAN}

\author{
Yves GUIVARC'H \\ (Collaboration with J.C. TAYLOR, L. JI)
}

\section{INTRODUCTION AND DESCRIPTION OF RESULTS.}

Consider a connected semi-simple real Lie group $G$ which has a finite center and no compact factors. One denotes by $A d$ the adjoint representation of $G$ in the Lie algebra $\mathscr{G}$ of $G$. Denote by $A$ a maximal connected subgroup of $G$ such that $A d A$ is diagonal, by $\alpha$ its Lie algebra, by $\|x\|^{2}=\operatorname{Tr}(a d x)^{2}$ the restriction of the Killing form to $a$. Let $K$ be a maximal compact subgroup of $G$ such that the centralizer $Z(A)$ of $A$ in $G$ can be written as $Z(A)=[K \cap Z(A)] A=M A$. Then the group action allow to construct from $x \rightarrow\|x\|^{2}$ a $G$-invariant Riemann metric on $X=G / K$ and this homogeneous space has the structure of a symmetric space on which $G$ acts by isometries [He]. The Laplace-Beltrami operator $L$ is then defined and one denotes by $e^{-\lambda_{0}}<1$ the spectral radius of $e^{L}$ in $\mathbb{L}^{2}(X)$. The resolvent $(D-\lambda I)^{-1}$ of $D=-L$ defines for $\lambda \leq \lambda_{0}$ a Green kernel $\left(G_{\lambda}(x, y)\right)$ which is a positive and symmetric $C^{\infty}$ function outside the diagonal of $X \times X$ such that $(D-\lambda I) G_{\lambda}$ is the Dirac kernel.

It is a classical fact of potential theory that the form of the $\lambda$-eigenfunctions of $D$ $\left(\lambda \leq \lambda_{0}\right)$ is strongly related to the behaviour at infinity of $G_{\lambda}$. The Martin kernel $K^{\lambda}(x, y)=\frac{G_{\lambda}(x, y)}{G_{\lambda}(0, y)}$, where 0 is the point of $X$ defined by $K$, satisfies uniform Harnack inequalities, hence the set of functions $K^{\lambda}(., y)$ is relatively compact $(y \in X)$. In the topology of uniform convergence on compact sets the cluster values of $K^{\lambda}(., y)$ when $y$ tends to infinity form a compact metric $G$-space of normalised $\lambda$-eigenfunctions which will be denoted $\partial m^{\lambda}$. In terms of $K^{\lambda}$ one can define a natural topology on $m^{\lambda}=X \cup \partial m^{\lambda}$ such that $X$ is homeomorphically embedded in $m^{\lambda}, K^{\lambda}(., y)$ extends continously to $m^{\lambda}$ and the closure of $X$ is $m^{\lambda}$. The function $\theta^{\lambda}$ on $G \times \partial m^{\lambda}$ defined by

$$
\begin{aligned}
& \theta^{\lambda}(g, \mu)=\lim _{y \rightarrow \mu} \frac{G_{\lambda}\left(g^{-1}, 0, y\right)}{G_{\lambda}(0, y)} \text { satisfies the cocycle identity } \\
& \theta^{\lambda}(g h, \mu)=\theta_{\lambda}(g, h, \mu) \theta_{\lambda}(h, \mu) .
\end{aligned}
$$


Here the action $\left(S_{h}\right)$ of $h \in G$ on the function $\mu \in \partial \mathrm{m}^{\lambda}$ is the projective action :

$$
h \cdot \mu(x)=S_{h} \mu(x)=\frac{\mu\left(h^{-1} \cdot x\right)}{\mu\left(h^{-1} \cdot 0\right)} .
$$

The space $\mathrm{m}^{\lambda}$ is a $G$-space which is called the Martin compactification of $X$ and the subset $\partial \mathrm{m}^{\lambda}$ is the Martin boundary of $X$ with respect to $L$ [Do]. It is known that $\partial \mathrm{m}^{\lambda}$ always contains the set $\partial \mathrm{m}_{e}^{\lambda}$ of minimal $\lambda$-eigenfunctions but can be larger. In the situation considered here, it turns out that $\partial m^{\lambda}$ consists solely of minimal eigenfunctions if and only if $\operatorname{dim} \alpha=1$.

The problem considered in this paper is to identify the $G$-space $\mathrm{m}^{\lambda}=X \cup \partial \mathrm{m}^{\lambda}$ in geometrical terms and to calculate the cocycle $\theta^{\lambda}$ in terms of the geometry of $X$ and its natural boundaries. One recall that the set of minimal eigenfunctions was determined in [Ka]. For a short proof of this, see [Gu2] and for a general approach to the calculation of minimal eigenfunctions see [Fu2]. Hence, the problem can be formally reduced to the description of the integral representation of limiting functions in terms of minimals, because the set of positive $\lambda$-eigenfunctions is a Choquet simplex [Cho]. Because one is concerned with the space $X=G / K$ and the groups of isometries of $X$ one can suppose $G$ is the set of real points in a semi-simple algebraic group $G_{a}$ defined over $\mathbb{R}$. The results can be conveniently expressed in terms of this explicit algebraic structure and the associated spaces of measures or directions.

The relation of this analytical problem with algebraic notions comes partly from the Harnack inequality. This inequality implies relative compactness of the projective $G$-orbits of eigenfunctions in the relevant functional spaces ; for special eigenfunctions this compactness reduces to the finite dimensionnality and projective character of the corresponding orbit closures.

In order to describe the answer, one has to consider the variety $F$ of minimal parabolic subgroups of $G$ [Bor] as well as the structure on the sphere at infinity of $X$ defined by the Weyl chambers. If $B$ is the set of real points of a minimal parabolic subgroup of $G_{a}$ defined over $\mathbb{R}$, the space $\mathscr{F}=G / B$ is a compact homogeneous space called the Furstenberg boundary of $X$ (or $G$ ). There exist on F a unique $K$-invariant probability measure $m$ and one considers the Poisson kernel $P(g, b)=\frac{d g \cdot m}{d m}(b)(g \in G, b \in \mathscr{F})$. The bounded solutions of the equation $L f$ $=0$ are then given by Poisson integrals

$$
f(g .0)=\int P(g, b) \hat{f}(b) d m(b)
$$


with $\hat{f} \in \mathbb{L}^{\infty}(m)$ [Fu1]. The space $X$ is naturally embedded in the space $\mathscr{P}(\mathscr{F})$ of probability measures on $\mathscr{F}$ via the map $i: i(g, 0)=g . m$. The space $\mathscr{P}(\mathscr{F})$ is a compact metrisable $G$-space with respect to the weak topology. The closure $i \overline{(X)}$ of $i(X)$ in $\mathscr{P}(\mathscr{F})$ defines a compactification of $X[\mathrm{Fu} 1, \mathrm{Gl}]$. This compactification turns out [Moo1] to be isomorphic to the maximal Satake-Furstenberg compactification $\bar{X}^{S F}$ of $X$ (or $G$ ) originally defined in terms of quadratic forms by I Satake [Sa]. The boundary behaviour of harmonic functions in this compactification has already been considered in [Ko] making use of the various group decompositions which occur in this paper.

Furthermore these decompositions and the families of measures which occur here play also an important role in the study of Lyapunoff exponents for product of random matrices [Gu-Ra1,2]. Closely related questions were already considered by E.B. Dynkin in the case of $G=S \ell(n, \mathbb{C})$ [Dy2]. The well known Cartan conical compactification $\bar{X}^{C}$ of $X$ as a Cartan-Hadamard manifold [C] plays also an important role here. If the rank of $G$ is not one, the compactification $\bar{X}^{C}$ is different from $\bar{X} S F$. One proves in this paper that the $G$ space $m^{\lambda_{0}}$ is isomorphic to $\bar{X} S F$. It is also shown that, if $\lambda<\lambda_{0}$, the $G$-space $m^{\lambda}$ is the smallest compactification of $X$ which dominates $\bar{X}^{C}$ and $\bar{X} S F$. Moreover the cocycle $\theta^{\lambda}$ on $G \times m^{\lambda}$ is calculated in terms of the Poisson kernel and the Busemann function [Bal-Gr-Sc].

From the results it will be clear that $\partial \mathrm{m}^{\lambda}$ has the structure of a topological manifold of dimension $\operatorname{dim} X-1, m^{\lambda}=X \cup \partial m^{\lambda}$ is a topological manifold with boundary $\partial \mathrm{m}^{\lambda}$ and $\partial \mathrm{m}_{e}^{\lambda}$ is a closed subset of full dimension, thereby verifying a conjecture of E.B. Dynkin [Dy1]. The parametrization of $\partial m_{e}^{\lambda}$ gives a natural embedding of $\partial \mathrm{m}_{e}^{\lambda}$ into $\bar{X}^{C} \times \mathscr{F} \subset \bar{X} C \times \bar{X}^{S F}$ but the corresponding maps into $\bar{X}^{C}$ and $\bar{X} S F$ are not injective hence the compactifications $\bar{X}^{C}$ and $\bar{X} S F$ are simultaneously needed to describe $\mathrm{m}^{\lambda}$.

In order to describe $m^{\lambda}, \theta^{\lambda}$ it is natural to embedd $X$ in the space $\mathscr{I}$ of closed subgroups of $G$ via the map $i^{\prime}$ defined by $i^{\prime}(g .0)=g K g^{-1}$ and to use the invariance properties of the limiting functions of $K^{\lambda}(., y)$. In particular the $G$ space $\bar{X} S F$ turns out to be isomorphic to the closure $\overline{i^{\prime}(X)}$ of $i^{\prime}(X)=\mathscr{S}_{0}$ in $\mathscr{S}$ with respect to the natural topology [Bou] (Hausdorff convergence on compact sets). The space $\overline{\mathscr{S}}_{0}=i^{\prime} \overline{(X)}$ can be characterized as the space of maximal distal subgroups of $G$ in the adjoint action [see I] and every element of $\overline{\mathscr{S}}_{0}$ has a 
unique fixed point in $\bar{X} S F$. This gives an answer to a question raised by $H$. Furstenberg about a possible unified conjugacy theorem valid for maximal compact subgroups and maximal amenable subgroups. Here the emphasis is on "distal subgroups" and the answer can be extended to the situation of semisimple groups defined over local fields. This is strongly related to [M002] but the approach is different. If $D \in \overline{\mathscr{Y}}_{0}$ is fixed, there exist a unique normalised $\lambda_{0}$-eigenfunction $h^{D}$ which is invariant under left translations by $D$. Then, if the sequence $y_{n}=g_{n}, 0 \in X$ is such that $g_{n} K g_{n}^{-1}$ converges to $D \in \overline{\mathscr{S}}_{0}$, the Martin kernel $K^{\lambda_{0}}\left(., y_{n}\right)$ converges toward the function $h^{D}$. Hence $\theta^{\lambda_{0}}(g, D)=h_{D}\left(g^{-1} .0\right)$ if $D \in \overline{\mathscr{S}}_{0}$. It turns out that the subgroup $D$ is contained in a parabolic subgroup $P(D)$ which is minimum for this property and the set of minimal parabolics contained in $P(D)$ is a fiber of $\mathscr{F}$ over $G / P(D)$. Recall [Ka, Gu2] that the minimal $\lambda_{0}$ - eigenfunctions are given by the square root of the Poisson kernel :

$$
h_{b}(g)=\left(\frac{d g \cdot m}{d m}\right)^{1 / 2}(b) \text {. }
$$

The function $h^{D}$ can then be uniquely expressed in terms of minimal eigenfunctions as :

$$
h^{D}(g)=\int\left(\frac{d g \cdot m}{d m}\right)^{1 / 2}(b) d m_{*}^{D}(b) .
$$

One denotes by $\sigma(g, b)$ the "Poisson cocycle" $\frac{d g^{-1} \cdot m}{d m}(b)$.

The probability measure $m_{*}^{D}$ is concentrated on the fiber of $\mathscr{F}$ corresponding to $P(D)$ and is uniquely determined by a $D$-invariance property. Clearly the map $D \rightarrow m_{*}^{D}$ is a $G$-equivariant isomorphism of $\overline{\mathscr{S}}_{0}$ into $\mathscr{P}(\mathscr{F})$. This gives an isomorphism between $\bar{X} S F$ and $m^{\lambda_{0}}$ in terms of integrals representations . When the parabolic subgroup $P(D)$ is fixed, the set of these measures (or functions) is a $P(D)$-space isomorphic to the symmetric space associated with the semi-simple part of $P(D)$. Hence the space $\overline{\mathscr{S}}_{0}$ is fibered over the set of parabolic subgroups and these fibers are symmetric spaces.

The conical compactification $\bar{X}^{C}$ also has already a natural identification with a functionnal space [Bal-Gro-Sc] : each point $z \in \partial \bar{X} C$ corresponds to the normalised Busemann function $A(x, z)=\lim _{y \rightarrow z} d(0, y)-d(x, y)$ where $d$ is the Riemann distance function on $X$. The function $\beta(g, z)=e^{A(g-1.0, z)}$ is a cocycle on $G \times \partial \bar{X} C$, called here the "Busemann cocycle". One denotes by $B(z)$ the stabiliser 
of $z \in \bar{X} C$ in $G$; if $z \in \partial \bar{X} C$, this is a parabolic subgroup of $G$. The space $\bar{X} C S F$ is defined to be the set of pairs $(D, x) \in \bar{X}^{S F} \times \bar{X}^{C}$ such that $D \subset B(x)$. If $j$ is the diagonal embedding of $X$ into $\bar{X} S F \times \bar{X}^{C}$ given by $j(x)=\left[i^{\prime}(x), x\right]$, it turns out that $\overline{j(X)}=X^{C S F}$ and this $G$-space is naturally isomorphic to the Martin compactification $\mathrm{m}^{\lambda}$ for $\lambda<\lambda_{0}$. The $\lambda$-eigenfunction $h_{z, D}^{\lambda}$ defined by $(D, z) \epsilon$ $\bar{X} C S F$ is obtained as follows : it is the product of $h^{D}(g)$ by a power of the Busemann cocycle $\beta(g, z)$; this power (in fact $-\sqrt{\lambda_{0}-\lambda}$ ) is determined by the condition that this product is a $\lambda$-eigenfunction. Hence :

$$
\theta^{\lambda}[g,(D, z)]=h^{D}\left(g^{-1}\right) \beta^{-\sqrt{\lambda_{0}-\lambda}}(g, z)
$$

In particular if $b \in g^{\prime}: \quad \theta^{\lambda}[g,(b, z)]=\sigma^{1 / 2}(g, b) \beta^{-\sqrt{\lambda_{0}-\lambda}}(g, z)$ is a minimal $\lambda$ eigenfunction.

The set of minimal eigenfunctions is the set of functions of the form

$$
h_{z, b}^{\lambda}(g)=\sigma^{1 / 2}\left(g^{-1}, b\right) \beta^{-\sqrt{\lambda_{0}-\lambda}}\left(g^{-1}, z\right),
$$

where $b \subset B(z)$. This condition defines a closed G-invariant subset of $\bar{X} C S F$ which parametrize the set of minimal $\lambda$-eigenfunctions and is denoted by $\bar{X}_{e}^{C S F}$.

In order to obtain $\bar{X} C S F$ from $\bar{X}$ C one has to imbed the set of general directions of $\bar{X}^{C}$ into $\bar{X}_{e}^{C S F}$ and then, "fill the holes" of $\bar{X}_{e}^{C S F}$ with the Satake-Furstenberg compactifications of symmetric spaces of smaller rank defined by the semisimple parts of parabolic subgroups (one for each singular direction). Moreover the subset of $\bar{X}^{C}$ consisting of the general directions $z$ such that $B(z)$ is a minimal parabolic subgroup is isomorphically embedded as an open and dense subset of $\bar{X}_{e}^{C S F}$. The complement in $\bar{X}^{C S F}$ is a fibered space over the set of singular directions. The relation of $m^{\lambda}$ with $m^{\lambda_{0}}$ is the same as in the classical potential Newtonian theory corresponding to the Euclidean situation where $G=\mathbb{R}^{d}$ and $L$ the ordinary Laplacian on $\mathbb{R}^{d}$; in this case $\lambda_{0}=0, \mathrm{~m}^{\lambda_{0}}$ is the one point and $m^{\lambda}$ is the sphere at infinity of $\mathbb{R}^{d}$. In the case of symmetric spaces of rank greater that one, the geometry of $\mathrm{m}^{\lambda_{0}}$ has a clear bearing in $m^{\lambda}$; this fact is hidden by some peculiarities in the Euclidean case as well as in the rank one case. 
The space $\bar{X} C S F$ has the natural factors $\bar{X} C$ and $\bar{X} S F$. The fiber of $\bar{X} C S F$ over $z \in$ $\partial \bar{X} C$ is the Satake-Furstenberg compactification of the semi-simple part of $B(z)$. In order to describe the fibers over $\bar{X} S F$, one observes that $\bar{X} C$ has a cellular decomposition of the following type : to each parabolic subgroup $P \subset G$ associate the "cell" $P_{\infty} \subset \partial \bar{X} C$ defined by

$$
P_{\infty}=\left\{z \in \partial \bar{X}^{C} ; B(z)=P\right\} .
$$

These cells are homeomorphic to the facets at infinity of the Cartan subalgebra a, defined by the root system of $G$. The fiber of $\bar{X} C S F$ over $D \in \bar{X} S F$ is the closed cell of $\bar{X} C$ defined by $P(D)$. From an algebraic point of view the space $\bar{X} C S F$ splits over the set of parabolic subgroups of $G$ : given a parabolic subgroup $P$, or a facet at infinity of $\bar{X}^{C}$ the set of pairs $(D, z)$ which appears above $P$ is the product of the Satake-Furstenberg compactification of the symmetric space associated to $P$ and of the Weyl chamber at infinity defined by $P$. The result for $m^{\lambda}\left(\lambda<\lambda_{0}\right)$ can be summarised in the following way : convergence of a sequence $y_{n}$ towards $z \in \partial \bar{X} C$ is not sufficient to insure the convergence of the Martin kernel $K^{\lambda}\left(., y_{n}\right)$; the stabilizer of $y_{n}$ must also converge in the space of closed subgroups of $G$; it turns out that this condition is sufficient. This is the reason for the splitting of $m \lambda$ as well as for the decomposition of the limiting function into a product of two functions defined by a pair of points in $\partial \bar{X} S F$ and $\partial \bar{X} C$. From the point of view of topological dynamics the orbit structures of $G$ in $\bar{X} C$ and $\bar{X} S F$ are easily understood. The first space is a union of compact homogeneous spaces of the form $\mathrm{G} / P$ where $P$ is a parabolic subgroup and this union is indexed by the points of the closed Weyl chamber at infinity. In the second space, the orbits of $G$ are locally closed and are "attracted" towards the unique compact one, which is isomorphic to $\mathscr{T}$. In particular $G$ is proximal on $\bar{X} S F \quad[G l, Z i]$ and conditionally proximal on $\bar{X} C$. The unique $G$-minimal set in $\bar{X} S F$ is isomorphic to $\mathscr{F}$. Generally speaking, here one has an example where some functionnal $G$-spaces which, a priori, are infinite dimensional spaces turn out to be close to $G$-spaces defined by rational actions of $G$ on projective manifolds in spite of the fact that $\bar{X} C$ and $\bar{X} S F$ have not such a structure.

Finally, from the probabilistic point of view one can draw weak corollaries : in each of these compactifications almost every trajectory of the Brownian motion defined by $L$ converges towards a random point of a special $G$-orbit in the boundary ; this orbit is isomorphic to $\mathscr{F}$. It follows from [Do], that if Brownian motion is conditionned with respect to the function $h_{z, D}^{\lambda}$ convergence a.e in 
$\bar{X} C S F$ takes place towards $(z, D) \in \bar{X} C S F$. On the other hand, conditioning with respect to a spherical function leads to convergence a.e, in $\bar{X} C$, towards a random point of a $G$-orbit in $\partial \bar{X} C$ associated with this spherical function. This $G$-orbit is a factor of the Furstenberg boundary. The situation is almost the same in $\bar{X} C S F$ but the $G$-orbit in $\partial \bar{X}^{C S F}$ is always isomorphic to $\mathscr{F}:$ it gives a more precise information. In terms of Liapunoff exponents and limits theorems quantitative statements can be formulated along the lines of $[\mathrm{Gu}$ Ra1].

For some classes of semi-simple groups the precise behaviour at infinity of the Green kernel had already been studied by several authors [Dy, Ol, No, Gi-Wo]. In the case of complex Lie groups it can be obtained in terms of special functions [Dy, No]. In the general case, asymptotics of the Green kernel $G_{\lambda}$ $\left(\lambda \neq \lambda_{0}\right)$ were stated in [Ol] for "generic directions". Among the pairs $(z, D)$ $\in \bar{X}^{C S F}$, such that $D=M N \in \bar{X}^{S F}$ contains a maximal unipotent subgroup $N$, the "generic directions" are those such that $z$ is defined by a non singular geodesic, hence $B(z)=B$ is a minimal parabolic subgroup and $z$ belongs to the open cell at infinity $P_{\infty}$, a case also coonsidered in [Boug] ; the non "generic directions" correspond to $z$ in the boundary of $P_{\infty}$, and $D=M N$. Hence, the structure of the full Martin compactification was unknown in general. Moreover the situation of trees and product of trees was considered in [Pi-Wo] ; in this paper this situation is also considered [see below]. Here the study of the case $\lambda=\lambda_{0}$ involves essentially symmetry and ground state considerations as in [Ta-Gu]. In general, $G_{\lambda}$ is comparable with a simple explicit kernel [An-Ji], for which the corresponding compactification can be calculated and shown to be the same as for $G_{\lambda}$.

The description given above suggests an extension to the situation where the Laplacian is replaced by a non degenerate $K$-bi-invariant probability measure $p$ on $G$. From the probabilistic point of view, this amount replacing Brownian motion on $X$ associated with $L$ by random walk on $G_{/ K}$ generated by $p$. In this context a larger class of locally compact groups can be considered. In particular one can consider the group $G=G_{F}$ of $F$-rationnal points of an algebraic semi-simple group $G_{a}$ defined and isotropic over a local field $F$; this include to some extent the situation of trees (which corresponds to rank one) [Ge]. In this more general context, the various objects considered above make sense and one describes briefly the relations between them. Here the group $G$ of $F$-rational points in $G_{a}$ is a locally compact and totally disconnected non amenable topological group. One fixes once for all a standard minimal $F$. 
parabolic subgroup $B_{a} \subset G_{a}$ and denotes $B=B_{a} \cap G$. One denotes by $K$ a maximal compact subgroup of $G$ which is supposed to be transitive on $G / B[\mathrm{Br}$ Ti]. The well-behaved (see II) probability measure $p$ is fixed and supposed to be $K$-bi-invariant. The homogeneous space $G_{/ B}$ plays in this situation the role of Furstenberg boundary and gives the bounded $p$-harmonic eigenfunctions. In this situation the number $e^{-\lambda_{0}}$ has to be replaced by the spectral radius $r_{0}$ of the convolution operator on $\mathbb{L}^{2}(X)$ defined by $p$. The Green kernel $G_{\lambda}$ has to be replaced by the potential kernel $V_{r}=\sum_{0}^{\infty} r^{-n} p^{n}\left(r \geq r_{0}\right)$.

The set of positive eigenfunctions of $p$ have the same form as above but the correspondance between eigenvalues and such eigenfunctions has to be naturally modified if $r>r_{0}$.

Once a certain conjugacy class of maximal compact subgroups is fixed, the $G$ spaces $\bar{X} S F$ and $\bar{X} C S F$ have analogous definitions in terms of measures on $\mathscr{F}=G / B$ and geodesics in the Bruhat-Tits building of $G$ : they are natural candidates for the Martin boundaries ; the cocycle $\theta^{r}$ has to be modified for $r>r_{0}$ in a natural way. In this context, the answer to the basic questions of this paper are shown to be the same as for the real field in the special case $r=r_{0}, G_{a}$ simply connected and split over $F$. The space $\bar{X} S F$ is the closure, in the space of closed subgroups of $G$, of the $G$-orbit $\mathscr{S}_{0}$ of $K$. For $D \in \overline{\mathscr{S}}_{0}$, the function $h^{D}$ is well defined by $D$-invariance and gives the corresponding cocycle. Hence the corresponding Martin compactification is $\bar{X} S F$. The situation in the local field case is briefly described in part III. The proofs of the corresponding facts are parallel to the proofs given in the real field context. Closely related questions were asked in $[\mathrm{Ca}]$. For informations on the properties of semi-simple algebraic groups defined over a field $F$ one may refer to [Bor]. If $F$ is a local field, the relevant properties of the locally compact group of $F$-rational points of $G_{a}$ can be found in [Mar, chap. I].

The extension of the real field situation to the more general situation of local fields is formulated as conjectures in parts III and IV

In order to illustrate the above discussion, one consider the special case $G=S \ell(d, \mathbb{R})$ of unimodular matrices of dimension $d$. In this case, one can take $A$ to be the set of diagonal matrices with positive coefficients $a=\operatorname{diag}\left(\lambda_{1}, \lambda_{2}, \ldots\right.$, $\left.\lambda_{d}\right)$ with $\lambda_{i}>0, \lambda_{1} \lambda_{2} \ldots \lambda_{d}=1$. A closed Weyl chamber $a^{-}+$is defined by the conditions $\mu_{1} \geq \mu_{2} \geq \ldots \geq \mu_{d}, \mu_{1}+\mu_{2}+\ldots+\mu_{d}=0$, one has $\bar{A}^{+}=\exp \left(\bar{a}^{+}\right)$and the simple roots are given by $\alpha_{i}=\log \lambda_{i+1}-\log \lambda_{i}$. 
The unipotent group $N$ is the set of strictly upper triangular matrices. The maximal compact subgroup $K$ is $K=S O(d)$, the rotation group with respect to the euclidean norm on $\mathbb{R}^{d}$.

In this case $\mathscr{F}$ is the space of complete flags in $\mathbb{R}^{d}$. A subset $I \subset\left\{\alpha_{1}, \alpha_{2}, \ldots, \alpha_{d}\right\}$ defines a partition of $[1,2, \ldots, d]$ again denoted by $I=\left(I_{1}, I_{2}, \ldots, I_{k}\right)$. Then $A(I)$ is the set of diagonal matrices $a=\operatorname{diag}\left(\lambda_{1}, \lambda_{2}, \ldots, \lambda_{d}\right)$ such that $\lambda_{i}=\lambda_{j}$ when the pair $(i, j)$ is contained in an interval of the partition. The parabolic subgroup $P(I)$ is the set of upper triangular matrices with square blocks of the above type along the diagonal. The corresponding factor $\mathrm{G} / P(I)$ is the space of incomplete flags of type $I=\left(I_{1}, I_{2}, \ldots, I_{k}\right)$; a typical flag of type $I$ is given by the sequence of subspaces

$$
V_{1} \subset V_{2} \subset \ldots . \subset V_{k}=\mathbb{R}^{d}
$$

with $V_{j}=\underset{i \in I_{j}^{\prime}}{\bigoplus} R e_{i}, I_{j}^{\prime}=I_{1} \cup I_{2} \cup \ldots \cup I_{j}$.

The fiber above a typical incomplete flag $d$ (of type I) is the set of complete flags $b$ which refine $d$, and the natural measure $m_{d}$ on such a fiber is the probability measure which is invariant under the rotations which preserve $d$. More generally the image of such a pair $\left(d, m_{d}\right)$ under a projective transformation will be called a measured flag. Then the $G$-space $\bar{X} S F$ can be identified with the $G$-space of measured flags [Gu, Ra1]. The closed subgroup $D^{I}$ associated with the typical measured flag $\left(d_{I}, m_{I}\right)$ is the subgroup of $P(I)$ with orthogonal blocks along the diagonal and the stabiliser of $\left(d_{I}, m_{I}\right)$ is the subgroup $R^{I} \subset P(I)$ defined by the weaker constraint of similitude instead of orthogonality.

A sequence $y_{n} \in X$ converges to $\left(d_{I}, m_{I}\right)$ if one can write $y_{n}=\varepsilon_{n} a_{n} .0$ with $\lim _{n} \varepsilon_{n}=I d, a_{n}=\operatorname{diag}\left(\lambda_{n}^{1}, \lambda_{n}^{2}, \ldots, \lambda_{n}^{d}\right) \in A(I) \cap \bar{A}^{+}, \lim _{n} \frac{\lambda_{n}^{i}}{\lambda_{n}^{i+1}}=+\infty$ if $(i, i+1)$ is not contained in an interval of the $I$-partition.

The space $\partial \bar{X}^{C}$ can be described as the set of directions in $X$. Such a typical direction $v \in \partial \bar{X} C$ is given by a vector $v=\operatorname{diag}\left(u_{1}, u_{2}, \ldots, u_{d}\right) \in \overline{\mathrm{a}}^{+}$with $u_{1} \geq u_{2} \geq \ldots$ $\geq u_{d},\|u\|^{2}=\sum_{i=1}^{d} u_{i}^{2}=1$. The sequence $y_{n} \in X$ converges to $v_{\infty} \in \overline{\mathrm{a}}_{\infty}^{+} \subset \partial \bar{X}^{C}$ if one can write $y_{n}=k_{n} a_{n} .0$ with :

$$
a_{n} \in \bar{A}^{+}, \lim _{n} \frac{\log a_{n}}{\left|\log a_{n}\right|}=v \quad \lim _{n} k_{n}=k \text { and } \operatorname{Ad} k(v)=v .
$$


A general direction is obtained from $v_{\infty}$ by the action of $K$. These directions form the sphere at infinity $\partial \bar{X} C$ of $X=G / K$.

Such a direction $z$ is defined by a pair $\left(k, v_{\infty}\right)$ with $k \in K, v_{\infty} \in \overline{\mathrm{a}}_{\infty}^{+}$and $z=k \cdot v_{\infty}$.

The complete flag $b$ associated to $k$ is given by a sequence of multivectors $\left(b_{1}, b_{2}\right.$ $\left., \ldots, b_{d-1}\right)$ with $b_{j+1}=b_{j} \wedge x_{j},\left\|b_{j}\right\|=1$; the decomposable multivector $b_{j}$ defines a $j$ dimensional subspace $V_{j}$ and one has $V_{1} \subset V_{2} \subset \ldots \subset V_{d-1} \subset V_{d}=\mathbb{R}^{d}$. With these notations, the Busemann cocycle $\beta(g, z)$ is given by $\beta(g, z)=\prod_{i=1}^{d-1} \mid g b_{i} \|^{u_{i}-u_{i+1}}$ where $z=k . v_{\infty}, v=\left(u_{1}, u_{2}, \ldots, u_{d}\right) \in \overline{\mathrm{a}}^{+} \quad\|v\|^{2}=\sum_{i=1}^{d}\left|u_{i}\right|^{2}=2 d$.

The Poisson kernel is given by

$$
P\left(g^{-1}, b\right)=\prod_{i=1}^{d-1}\left\|g b_{i}\right\|^{-2}
$$

Hence, if one denotes $\sigma_{i}(g, b)=\left\|g b_{i}\right\|$, the minimal $\lambda$-eigenfunctions are given by

$$
h_{z, b}^{\lambda}(g)=\prod_{i=1}^{d-1} \sigma_{i}^{-1-\left(u_{i}-u_{i+1}\right)}\left(g^{-1}, b\right)
$$

with $u_{i}-u_{i+1} \geq 0(1 \leq i \leq d-1)$ and $\sum_{i=1}^{d} u_{i}^{2}=2 d\left(\lambda_{0}-\lambda\right)$,

$v=\operatorname{diag}\left(\frac{u_{1}}{|u|}, \ldots, \frac{u_{d}}{|u|}\right), \sum_{i=1}^{d} u_{i}=0$ and $z=k \cdot v_{\infty}$

If $y_{n} \in X$ converges in $\bar{X}^{C}$ to $z$ and in $\bar{X} S F$ to $b \in \mathscr{F}$, then the Martin kernel $K^{\lambda}\left(., y_{n}\right)$ converges to $h_{z, b}^{\lambda}$. If, instead of converging to $b, y_{n}$ converges to a typical measured flag $\left(d, m_{d}\right)$, then the Martin kernel converges to the non minimal eigenfunction $\int h_{z, b}^{\lambda}(g) d m_{d}(b)$.

The structure of this paper is as follows. To begin with, one gives some preliminary informations of a group-theoretical character. Then in a first part one describe the space $\bar{X} S F$ in terms of the space of closed subgroups of $G$; this characterisation is useful later on. In a second part one calculates $m^{\lambda_{0}}$ in the context of a random walk using the "ground state" characterisation of the functions $h^{D}$. In a third part one uses the above results to describe briefly the situation in the case of semi-simple groups defined over local fields. In the last part one calculates $m^{\lambda}$; in the spirit of part II one tries to characterize the 
Martin limits by their extremal and group-invariance properties, rather than just giving a calculation.

In view of the results and techniques of this paper it is natural to conjecture that, if $K$-bi-invariance of the probability measure $p$ is not assumed, the Martin compactifications of $G$, with respect to $p$ will remain the same and the new family of cocycles $\theta^{\lambda}$ will be obtained from the canonical family described in this paper by cohomology. The stability of the Martin compactification is well known in the case of $G=\mathbb{R}^{d}[\mathrm{Bab} 2]$.

The main results of this paper are part of a joint work with Lizhen Ji and J.C. Taylor (to appear); a preliminary version has appeared in Comptes Rendus [Gu-Ji- Ta]. The emphasis here is on the intrinsic approach and the random walk point of view in such a way that the situation of semi-simple groups defined over a local field could be simultaneously considered. The details will appear in another paper.

Thanks are due to M. Babillot and J.P. Anker for essential informations about the Green kernel. The first author is also grateful to G.A. Margulis who brought the question to his attention.

SOME GROUP-THEORETICAL PRELIMINARIES.

One describe here some known preliminary group-theoretical results.

\section{1) Parabolic subgroups and Furstenberg boundary.}

Consider a maximal subgroup $A$ of the semi-simple group $G$ such that $A d A$ is diagonal and $A$ is isomorphic to the multiplicative group $\left(\mathbb{R}^{+}\right)$. Consider its Lie algebra $\alpha \subset \mathscr{G}$, the set of roots $\Sigma$ of $\alpha$ in $\mathscr{G}$ and the root spaces $\mathscr{S}_{\alpha} \subset \mathscr{G}(\alpha \in \Sigma)$ defined by

$$
\mathscr{S}_{\alpha}=\{x \in \mathscr{G} ;[a, x]=\alpha(a) x \quad \forall a \in \alpha\}
$$

Once a Weyl chamber $\alpha^{+}$has been choosed in $\alpha$, the set $\Sigma^{+}$of positive roots is fixed and a system of simple roots $\Delta=\left(\alpha_{1}, \alpha_{2}, \ldots, \alpha_{r}\right) \subset \Sigma^{+}$is defined. One can define the nilpotent subalgebra

$$
\mathcal{N}=\underset{\alpha \in \Sigma^{+}}{\oplus} \mathscr{G}_{\alpha}
$$

and consider the corresponding unipotent connected subgroup $N \subset G$. The centraliser $Z(A)$ of $A$ is the product of its maximal compact subgroup $M$ by $A$. Hence $N$ is normalised by $Z(A)$ and the subgroup $B=Z(A) N$ is the semidirect product of $N$ and $Z(A)$. 
In analogy with the theory of semi-simple algebraic groups [Bor], $B$ will be called a standard minimal parabolic subgroup. More precisely, if $G_{a}$ is an algebraic group and its Lie algebra $\mathscr{G}_{a}$ is isomorphic to the complexification of $\mathscr{G}$ one can choose a $\mathbb{R}$-structure on $G$ such that, up to finite covering, $G$ is the connected component of the subgroup of real points of such a group $G_{a}$; then there exist in $G_{a}$ a unique standard minimal parabolic subgroup $B_{a}$ such that $B=G \cap B_{a}$. Moreover $B_{a}$ is the Zariski closure of $B$ in $G_{a}$.

Definition 1. A subgroup of $G$ will be said to be parabolic if it contains a conjugate of $B$. The homogeneous space of minimal parabolic subgroups will be called the Furstenberg boundary of $G$. It will be denoted $\mathscr{F}=G / B$.

The algebraic theory of parabolic subgroups have a complete analytic counterpart in the context of connected real semi-simple groups [Bor]. In particular there is only a finite number of such subgroups, up to conjugacy, and they correspond to the finite list of $G$-equivariant factors of $\mathscr{F}=G_{/ M A N}$. The homogeneous space $\mathscr{F}$ appears in [Fu 1] in the context of bounded harmonic functions ; it is the set of real points of a projective variety, defined over $\mathbb{R}$, on which $G$ acts by projective transformations.

\section{2) Standard parabolic subgroups.}

In order to describe more explicitely the parabolic subgroups, one consider "standard parabolic subgroups" associated to a subset $I \subset\left\{\alpha_{1}, \alpha_{2}, \ldots, \alpha_{r}\right\}$, one denotes by $[I]$ the set of positive roots which are linear combinations of elements of $E$ and write []$^{\prime}=\Sigma^{+}-[I]$. One consider the subalgebras

$$
\begin{aligned}
& \alpha(I)=\{x \in \alpha ; \alpha(x)=0 \quad \forall \alpha \in I\} \\
& \mathcal{N}(I)=\underset{\alpha \in[]^{\prime}}{\oplus} \mathscr{G}_{\alpha} \\
& \mathcal{N}^{I}=\underset{\alpha \in[\Pi]}{\oplus} \mathscr{G}_{\alpha} .
\end{aligned}
$$

One has $\mathcal{N}=\mathcal{N}^{I} \oplus \mathcal{N}(I),\left[\mathcal{N}(I), \mathcal{N}^{I}\right] \subset \mathcal{N}(I)$. One denotes by $\varphi I$ the sum of the subspaces $\mathscr{S}_{\alpha}+\left[\mathscr{S}_{\alpha}, \mathscr{S}_{-\alpha}\right]$ where $\pm \alpha$ varies over [I].

One consider the corresponding connected subgroups $A(I), N(I), N I$. The subspace $\mathscr{C} I$ is a semi-simple subalgebra and the corresponding connected subgroup will be denoted $G^{I}$.

The centralizer $Z(I)$ of $A(I)$ in $G$ is reductive and the standard parabolic subgroup $P(I)$ is defined as $P(I)=Z(I) N$. It is clear that $N(I), A(I) N(I)$ are normal subgroups of $P(I)$ and the relation $Z(I) \cap N(I)=\{0\}$ implies that $P(I)$ is 
the semi-direct product of $Z(I)$ and the normal subgroup $N(I)$. Because each $\mathscr{S}_{\alpha}$ is invariant under the adjoint action of $M A$, one has for $g \in G^{I}, h \in M: h g h^{-1}$ $\in G^{I}$; it follows that $M(I)=G^{I} M$ is a closed subgroup of $G$. This implies that $Z(I)=M(I) A(I), N(I)$ is normal in $P(I)$ and $P(I)=M(I) A(I) N(I)$. More precisely $P(I)$ is the semi-direct product of its unipotent radical $N(I)$ by the Levi subgroup $M(I) A(I)$. This decomposition is called the Langlands decomposition of $P(I)$.

It is known that $A^{I}=G^{I} \cap A$ is a maximal diagonal subgroup of $G^{I}$ isomorphic to $\left(\mathbb{R}^{+}\right)^{8}$ where $s$ is the number of elements in $I$ [Bor]. Moreover the restrictions of the roots in $I$ to $A^{I}$ define a set of simple roots of $G^{I}$. It follows that if $a^{I}$ denotes the Lie algebra of $A^{I}$, then the corresponding Weyl chamber $(a I)^{+}$is the orthogonal projection of $\alpha^{+}$in $\alpha^{I}$ with respect to the Killing form of $\alpha$. Consider now the projection of $\mathscr{F}=G / B$ onto the homogeneous space $G / P(I)$ and the orbit $\mathscr{F}(I) \subset \mathscr{F}$ of the origin $\bar{e} \in \mathscr{F}(\bar{e}=\{P\})$ under $P(I)$. This is a compact set which may be identified with the homogeneous space $P(I) / B$. The fibers of the projection are exactly the translates of $\mathscr{F}(I)$ under $G$. As a $G^{I}$-space, $\mathscr{F}(I)$ is isomorphic to the Furstenberg boundary of $G^{I}$ as it is now shown as follows. The centraliser of $A^{I}$ in $G^{I}$ is contained in $M A$ because it centralise $A^{I}, A(I)$ and $A=A^{I} A(I)$. This centraliser is equal to $M A \cap G^{I}$ and the relation $G^{I} M \cap A=A^{I}$ implies

$$
M A \cap G^{I}=\left(M \cap G^{I}\right)\left(A \cap G^{I}\right)=M^{I} A^{I}
$$

where $M^{I}=M \cap G^{I}$.

On the other hand the subgroup $A(I) N(I)$ is normal in the stabiliser $B$ of $\bar{e}:$ it acts trivially on $\mathscr{F}(I)$. From the relation $P(I)=G^{I} M A(I) N(I)$ it follows that $G^{I}$ is transitive on $\mathscr{F}(I)$. From the relations explained above it follows that $G^{I} \cap M A N$ $=\left(G^{I} \cap M A\right)\left(G^{I} \cap N\right)=M^{I} A^{I} U^{I}$. So that one has the

Lemma 2. The fiber of the origin of $G / P(I)$ in $G / P$ is isomorphic, as a $G^{I}$-space, to the Furstenberg boundary of $G^{I}$. The subgroup $A(I) N(I)$ acts trivially on $P(I) / B$, so that the action of $P(I)$ on this fiber reduces to the action of $G^{I} M=M(I)$.

\section{3) Maximal compact subgroups and Iwasawa decompositions.}

Consider now a maximal compact subgroup $K$ containing $M$ and the Iwasawa decomposition $G=K A N$. Because $G$ permutes the fibers of $\mathscr{F}$ above $G / P(I), K$ is transitive on $\mathscr{F}$ and the stabiliser of $\mathscr{F}(I)$ is $P(I)$, the subgroup $K \cap P(I)$ has to be transitive on $\mathscr{F}(I)$. Denote $K_{0}^{I}=K \cap G^{I}, K^{I}=K \cap M(I)$ and observes that the action of $K \cap P(I)$ on $\mathscr{F}(I)$ reduces to the action of $K^{I}: K^{I}$ is transitive on $\mathscr{F}(I)$. 
From the formulae $K^{I}=K_{0}^{I} M$ and $\mathscr{F}(I)=K^{I} \cdot \bar{e}$ one gets $\mathscr{F}(I)=K_{0}^{I} \cdot \bar{e}$ and $G^{I}=K_{0}^{I}$ $A^{I} U^{I}, P(I)=K_{0}^{I} M A N$. The formula $\mathscr{F}(I)=K_{0}^{I} \cdot \bar{e}$ implies that $K_{0}^{I}$ or $K^{I}$ leaves invariant a unique probability measure $m_{I}$ on $\mathscr{F}(I)$. In particular if $I=\phi$, then $m=m_{\phi}$ is the $K$-invariant measure on $\mathscr{T}$. The formula $G^{I}=K_{0}^{I} A^{I} N^{I}$ implies that $K_{0}^{I}, K^{I}$ are the stabilisers of $m_{I}$ in $G^{I}, G^{I} M$ and are maximal compact subgroups of $G^{I}, G^{I} M$ respectively.

To summarise the above discussion one state the lemma :

Lemma 3. If $K_{0}^{I}=K \cap G^{I}, A^{I}=A \cap G^{I}, N^{I}=N \cap G^{I}$, the group $G^{I}$ admits the Iwasawa decomposition $G^{I}=K_{0}^{I} A^{I} N^{I}$. Furthermore the stabiliser of $m_{I}$ in $G^{I}$ is $K_{0}^{I}$ and $K_{0}^{I}$ is a maximal compact subgroup of $G^{I}$.

\section{4) Satake-Furstenberg compactification.}

Consider the space $\mathscr{P}(\mathscr{F})$ of probability measures on $\mathscr{F}$, endowed with the weak topology. This a metrisable compact $G$-space. The closure $\overline{G \cdot m}$ of the orbit $G . m$ of $m$ under $G$ in $\mathscr{P}(\mathscr{F})$ is compact and one consider the map $g \rightarrow g$. $m$ of $G$ into $\mathscr{P}(\mathscr{F})$; one denotes by $0=\{K\}$ the origin in the symmetric space $X=G / K$.

Definition 4. The space $\overline{G . m}$ with the embedding of $G_{/ K}$ into $\overline{G . m}$ given by $g$. $0 \rightarrow$ $g$. $m$ will be called the maximal Satake-Furstenberg compactification of $G / K$ and onedenotes $\overline{G \cdot m}=\bar{X} S F$.

Because the stabiliser of $m$ is $K$, this map is well defined and is a homeomorphism of $G_{/ K}$ onto $G$. m. This compactification of $X=G_{/ K}$ is shown in [Moo1] to be isomorphic to one of the compactifications originally, defined by Satake in terms of quadratic forms (see below). The definition in term of measures was given in [Fu 1] and extends naturally to the general case of a semi-simple group defined over a local field (see below).

If $\mathrm{a}^{+}$is the Weyl chamber in $\alpha$ defined by $\Delta$ and $A^{+}=\exp \left(\mathrm{a}^{+}\right)$one has [He] the polar decomposition of $G: G=K \bar{A}^{+} K$. The $K$-parts of the decomposition $g=k a k^{\prime}$ are not unique in general but $a$ is uniquely defined. In this way sequences in $G$ going to infinity can be reduced to sequences in $\bar{A}^{+}$and the following notion is very useful. 
Definition 5. The sequence $a_{n} \in A$ will be said to be I-canonical if $a_{n} \in \bar{A}^{+}, e^{\alpha}\left(a_{n}\right)=1$ when $\alpha \in\left[\Pi, \lim _{n} e^{\alpha}\left(a_{n}\right)=+\infty\right.$ when $\alpha \in[I]^{\prime}$.

Lemma 6. Suppose that the sequence $a_{n} \in A$ is I-canonical. Then the sequence of measures $_{n}$. $m$ converges to $m_{I}$.

Proof. Consider the unipotent subgroup $\check{N}(I)$ opposite to $N(I)$ : its Lie algebra is given by $\check{\mathcal{N}}(I)=\oplus \mathscr{G}_{-\alpha}$. Clearly, for $x \in \mathscr{G}_{\alpha}, a \in A$ one has $\alpha \in[]^{\prime}$

$$
(A d a)(x)=e^{-\alpha(\log a)} x .
$$

Because $a_{n}$ is I-canonical one has $\lim _{n} e^{-\alpha\left[\log a_{n}\right]}=0$ for $\alpha \in[]^{\prime}$ and one conclude :

$$
\begin{aligned}
& \forall x \in \check{\mathcal{N}}(I): \lim _{m}\left(A d a_{n}\right)(x)=0 \\
& \forall \eta \in \check{N}(I): \lim _{m} a_{n} \eta a_{n}^{-1}=e .
\end{aligned}
$$

Consider now the space $G / P(I)$ which is a factor of $\mathscr{F}$. From the Bruhat decomposition, it follows that $\breve{N}(I) B(I)$ is an open dense set of $G$, so that the orbit of the origin in $G / P(I)$ under $\check{N}(I)$ is open and dense. If this orbit is

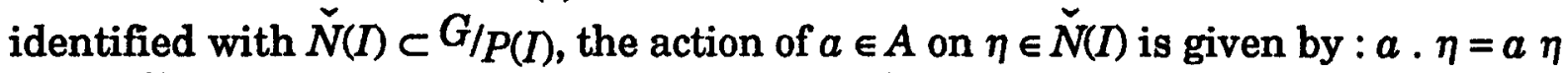
$a^{-1} \in \check{N}(I)$. It follows that, for every $\eta \in \check{N}(I) \subset G / P(I)$, the sequence $a_{n} . \eta$ converges to the origin. If $\vec{m}$ is the projection of $m$ onto $G / P(I)$, one obtain from dominated convergence, that the sequence of measures $a_{n} \cdot \bar{m}$ converges toward the Dirac measure at the origin. It follows that every cluster value of the sequence $a_{n}, m$ has a support contained in $P(I)_{/ P} \subset \mathcal{F}$. Because $a_{n}$ is $I$ canonical, $K^{I}$ commute with $a_{n}$ it follows that such a cluster value is $K^{I}$ invariant. There is only one probability measure on $P(I) / P$ with is $K^{I}$-invariant : this is the measure $m_{I}$. Finally

$$
\lim _{n} a_{n} \cdot m=m_{I} \text {. }
$$

Proposition 7.G.m is the disjoint union of the orbits of the measures $m_{I}$.

Proof. If $g_{n} \in G$ is such that the sequence $g_{n}$. $m$ converges one can suppose that $g_{n}=k_{n} a_{n} k_{n}^{\prime}$ with $a_{n} I$-canonical $\lim _{n} k_{n}^{\prime}=k^{\prime}, \lim _{n} k_{n}=k$. It follows from lemma $6: \lim _{n} g_{n} . m=k$. $m_{I}$. Hence

$$
\overline{G \cdot m}=\cup_{I} G \cdot m_{I} .
$$


Suppose $G \cdot m_{I} \cap G \cdot m_{I^{\prime}} \neq \phi$; then for some $g: g \cdot m_{I}=m_{I^{\prime}}$, and the supports of these measures are equal : $g P(I)=P\left(I^{\prime}\right)$. Because the fibers $g P(I)$ of $\mathscr{F}$ above $\mathrm{G} / P(I)$ are either disjoint or equal one has $g P(I)=P(I)=P\left(I^{\prime}\right), I=I^{\prime} \quad m_{I}=m_{I^{\prime}}$, $G \cdot m_{I}=G \cdot m_{I^{\prime}}$.

In order to explain the relation between the above compactification and the original construction of I. Satake [Sa1] consider a complex faithfull irreducible representation $\tau$ of $G$ such that, if $\theta$ denotes the Cartan involution of $G$ one has :

$$
\tau[\theta(g)]=t \overline{\tau[\theta(g)]^{-1}}
$$

Consider also the embedding of $\mathrm{G} / K$ into the space $\mathscr{X}$ of positive hermitian matrices given by $\tau(g K)=\tau(g) t \tau(g)$. If $P(\mathscr{H})$ is the real projective space of $\mathscr{X}$ one has an imbedding

$$
\tau: X \longrightarrow P(\mathscr{H}) .
$$

The closure $\overline{\tau(X)}$ is the Satake compactification of $X$ associated with the representation $\tau$, denoted by $\bar{X}_{\tau}^{S}$.

The compactification $\bar{X}_{\tau}^{S}$ is uniquely determined by the restricted dominant weight of the representation $\tau$. If the stabiliser of the dominant weight vector of $\tau$ is a minimal standard parabolic subgroup, then the compactification $\bar{X}_{\tau}^{S}=\bar{X}_{\max }^{S}$ is maximal. This is the only one which is used here. It is proved in [Moo 1,2] that the stabiliser of $q \in \bar{X}_{\max }^{S}$ is a conjugate of a group of the form

for some $I$.

$$
R^{I}=K^{I} A(I) N(I)
$$

Such a group stabilises a unique measure on $\mathscr{F}:$ this is the measure $m_{I}$. The correspondance between $\bar{X}_{\max }^{S}$ and $\bar{X} S F$ replaces $q \in \bar{X}_{\max }^{S}$ by the unique probability measure on $\mathscr{F}$ which is invariant under the action of the stabiliser of $q$. Instead of these results one uses here only the above definition in terms of measures. This language extends to semi-simple groups over local fields.

A continuous function on a locally compact group $H$ will be called an exponential if it is a homomorphism of $H$ into the multiplicative group of positive real numbers. 
The normaliser of $D^{I}=K^{I} N(I)$ is $R^{I}$ and $D^{I}$ is the subgroup of $R^{I}$ where the exponentials of $R^{I}$ are trivial. It will be clear below that the compactification $\bar{X}_{\max }^{S}$ is isomorphic to the space of subgroups of $G$ conjugate to some $D^{I}$ endowed with the natural topology.

\section{I - LIMITS OF MAXIMAL COMPACT SUBGROUPS AND THE MAXIMAL SATAKE- FURSTENBERG COMPACTIFICATION.}

One gives here a new characterisation of the maximal Satake-Furstenberg compactification. Hence the proofs are independent of the end of paragraph 4 above.

One consider the space $\mathscr{S}$ of closed subgroups of $G$ endowed with the topology of Hausdorff convergence on compact subsets of $G$. For $G$ locally compact this a metrizable compact space on which $G$ acts continuously by conjugation [Bou]. In the simplest non trivial example $G=\mathbb{R}$ one consider the family of subgroups $H_{a}=a \mathbb{Z}(a \in \mathbb{R})$. In this case $\lim _{a \rightarrow b} H_{a}=H_{b}$ if $b \neq 0, \lim _{|a| \rightarrow \infty} H_{a}=\{0\}, \lim _{a \rightarrow 0} H_{a}=\mathbb{R}$.

In order to give another example, in the spirit of this paper, one take $G$ equal to the additive group of a finite dimensional vector space $V$ over a locally compact field. Then the projective space $P(V)$ of $V$, is clearly a compact subset of the set of closed subgroups of $V$.

Here one consider the maximal compact subgroup $K$ and its orbit $\mathscr{S}_{0}=\left\{\mathrm{g} K \mathrm{~g}^{-1}\right.$; $g \in G$ ). In this case the elements of the closure $\overline{\mathscr{S}}_{0}$ in $\mathscr{S}$ can be interpreted as generalised horocycles. In the case $G=S \ell(2, R), X=G / K$ is the Poincaré disk of center $0=\{K\}$ and one can associate to a closed subgroup $H \subset G$, the orbit $H .0 \subset X$. Let $N$ denote the maximal unipotent subgroup of upper-triangular matrices and $M$ the set of diagonal matrice of the form $\operatorname{diag}\left(\varepsilon, \varepsilon^{\prime}\right)$ with $\varepsilon, \varepsilon^{\prime}= \pm 1$. Then it can be seen that the limits of compact subgroups are compacts subgroups or conjugates of the subgroup $M N$. Under the map of $G$ into $X$ considered above the set $g K^{-1}$ projects on $X$ as a circle $g K^{-1} .0$ of center $g^{-1} .0$ containing 0 . A sequence of circles either converge to such a circle, or converge towards an horicycle containing 0 and corresponding to a conjugate of $M N$. In order to calculate in general the closure $\overline{\mathscr{S}}_{0}$, one introduce some notations and lemmas. The corresponding analysis has an elementary character and can be carried over to other situations. Closely related questions have been considered in [Moo2]. 
Definition 1. If $V$ is a finite dimensional vector space and $H$ a closed subgroup of $G \ell(V)$ then $H$ is said to act distally on $V$ if for every $h \in H$, the eigenvalues of $h$ are numbers of modulus one. In particular $H \subset G$ is said to be distal if Ad $H$ acts distally on the Lie algebra of $G$.

It is known [Co-Gu], but not used for the main results that $H$ acts distally on $V$ if and only if for every $v \in V, v \neq 0$ the orbit closure of $H . v$ does not contain 0 . This is the reason for the word distal borrowed from topological dynamics. Clearly a compact subgroup and a unipotent subgroup are both distal. In order to prove proposition 8 below one needs some lemmas.

Lemma 2. Ev ery limit of distal subgroups is distal.

Proof. If $\lim _{n} H_{n}=H$, then for every $g \in H$ there exist a sequence $g_{n} \in H_{n}$ with $g=\lim g_{n}{ }^{n}, A d g=\lim A d g_{n}$. Since the spectrum of $A d g$ is the limit of the spectrum of $A d g_{n}$ and the unit circle is closed, the lemma follows.

Lemma 3. Suppose $J_{n} \subset G$ is sequence of closed subgroups, $\mu_{n}$ is a sequence of probability measures on F such that

a) $\mu_{n}$ is $J_{n}$-invariant.

b) $\lim \mu_{n}=\mu$ exist.

c) $\lim _{n}^{n} J_{n}=J$ exist.

Then $\mu$ is J-invariant.

Proof. As above consider $g \in J$ and $g_{n} \in J_{n}$ with $\lim _{n} g_{n}=g$. If $\varphi$ is a continuous function on $\mathscr{F}$, then the sequence of functions $\varphi\left(g_{n} . x\right)$ converges uniformly to $\varphi(g x)$. Hence, $g_{n} \cdot \mu_{n}(\varphi)$ converges to $g \cdot \mu(\varphi)$. This shows the weak convergence of $g_{n} \cdot \mu_{n}$ to $g \cdot \mu$ and implies the $F$-invariance of $\mu$.

Lemma 4. Suppose that the sequence $a_{n} \in A$ is I-canonical. Then the sequence of measures $a_{n} . m$ converges to $m_{I}$.

Proof. See the above paragraph.

Lemma 5. Suppose that the sequence $a_{n}$ is I-canonical and the sequence of closed subgroups $a_{n} K a_{n}^{-1}$ converge to $D \in \mathscr{Y}$. Then $D \supset K^{I} N(I)$.

Proof. Clearly, because $A(I)$ centralises $K^{I}$, one has $a_{n} K a_{n}^{-1} \supset K^{I}$. Denote by $y$ an element of $N(n)$ and observe that, because $a_{n}$ is $I$-canonical $\lim _{n} a_{n}^{-1} y a_{n}=e$. 
Using Iwasawa decomposition one can write $a_{n}^{-1} y a_{n}=k_{n} \alpha_{n} y_{n}^{\prime}$ with $k_{n} \in K$, $\alpha_{n} \in A, y_{n}^{\prime} \in \check{N}$ and observe that $\lim _{n} k_{n}=\lim _{n} \alpha_{n}=\lim _{n} y_{n}^{\prime}=e$ since $\lim _{n} a_{n} y_{n}^{\prime} a_{n}^{-1}$ $=e$. It follows that :

$$
\lim _{n} a_{n} k_{n} a_{n}^{-1}=\lim _{n} a_{n}\left(k_{n} \alpha_{n} y_{n}^{\prime}\right) a_{n}^{-1}=y .
$$

This proves that $\lim _{n} a_{n} K a_{n}^{-1} \supset N(I)$.

Lemma 6. The stabiliser of $m_{I}$ in $M(I)$ is compact.

Proof. One uses a typical contraction argument [Fu 1]. One knows that the homogeneous space $P(I) / B$ is the Furstenberg boundary of $M(I)$ or $G^{I}$, hence a projective variety. Consider an irreductible representation $\rho$ of $G^{I}$ into a finite dimensional vector space such that $P(I) / B$ is represented as a projective variety on which $G^{I}$ acts by projective transformations. If one considers a norm $\|$.$\| on$ $V$, it suffice to show that if the action of $\rho(g)\left(g \in G^{I}\right)$ onto this projective variety stabilise $m_{I}$, then $\|\rho(g)\|$ is bounded by a constant independent of $g$. If it not the case, then there exist a sequence $g_{n} \in G^{I}$ such that $\lim _{n}\left\|\rho\left(g_{n}\right)\right\|=+\infty$, hence $\lim _{n}$ $\operatorname{det} \frac{\rho\left(g_{n}\right)}{\left|\rho\left(g_{n}\right)\right|}=0$. The sequence of linear maps $u_{n}=\frac{\rho\left(g_{n}\right)}{\|\left(g_{n}\right) \mid}$ satisfies $\left\|u_{n}\right\|=1$, hence one can suppose by compactness of the unit ball, that $\lim u_{n}=u,|u|=1$, $\operatorname{det} u=0$. Hence one obtains that the measure $v=\lim _{n} u_{n} . m_{I}=u, m_{I}$ is concentrated on the union of two projective subspaces which corresponds to Im $u$ and Ker $u$ [see Fu 1]. But then the measure $m_{I}=\lim _{n} \rho\left(g_{n}\right) . m$ would be concentrated on the union of two projective subspaces. Hence a subgroup of $G^{I}$ of index at most two would leaves each of these subspaces invariant. Because $G^{I}$ is transitive on $P(I)_{B}$ and $\rho$ irreducible, this is impossible.

Remark. Only the facts that $v=m_{I}$ is not concentrated on the subspace corresponding to Ker $u$ and the stabiliser of the support of $v=m_{I}$ is irreducible have been used in this proof.

Lemma 7. The stabiliser of the measure $m_{I}$ in $G$ is $R^{I}=K^{I} A(I) N(I)$. The normaliser of $D^{I}=K^{I} N(I)$ is $R^{I}$.

Proof. The support of $m_{I}$ is $P(I) / B$. If $r$ stabilises $m_{I}, r$ also stabilises its support, so that $r . P(I) / B=P(I) / B$, hence $r \in P(I)$.

This proves that the stabiliser $L$ of $m_{I}$ is contained in $P(I)$. Observe that the relation $b A(I) N(I) b^{-1}=A(I) N(I) \subset B$ for every $b \in P(I)$ implies that $A(I) N(I)$ 
acts trivially on $P(I)_{B}$. Hence the subgroup $R^{I}=K^{I} A(I) N(I)$ stabilise $m_{I}$ and so : $R^{I} \subset L \subset P(I)$. On the other hand, from lemma 6, one knows that $L \cap M(I)$ is a compact subgroup. Because $K^{I}$ is maximal, as a compact subgroup of $M(I)$, and $L \cap M(I) \supset K^{I}$, one has $L \cap M(I)=K^{I}$. From the relation $R^{I} \subset L \subset P(I)=M(I) A(I)$ $N(I)$ one obtains $L=R I$.

Denote again by $L$ the normaliser of $D^{I}$. The subgroup $L$ normalises also the unipotent radical $N(I)$ of $D^{I}$. But the normaliser of $N(I)$ is $P(I)$ and this implies $L \subset P(I)$. Moreover $D^{I}$ leaves invariant a unique measure on $P(I) / B$ so that $L \subset P(I)$ leaves also invariant this measure $m_{I}: L \subset R^{I}$. Because $R^{I}$ normalises $D^{I}$ one has $L=R^{I}$.

Proposition 8. Suppose that $a_{n} \in A$ is I-canonical. Then the sequence of compact subgroups $a_{n} K a_{n}^{-1}$ converges to $D^{I}=K^{I} N(I)$.

Proof. Consider a convergent subsequence $D_{n}=a_{n} K a_{n}^{-1}$ with $\lim D_{n}=D$. The lemmas 3, 4, 5 above implies that $D \supset K^{I} N(I)$ and $D$ preserves $m_{I}$. Because the stabiliser of $m_{I}$ is $R^{I}=K^{I} A(I) N(I)$ one has $R^{I} \supset D \supset K^{I} N(I)$.

The first lemma implies that $D$ is distal and because, for $a \in A \quad a \neq e$ the action of $A d a^{n}$ on $N(I)$ is not distal, one has $D \cap A(I)=e$. The relation $R^{I}=K^{I} A(I) N(I)$ implies finally $D=K^{I} N(I)$. So that, in fact the whole sequence $a_{n} K a_{n}^{-1}$ converges to $D^{I}$.

Corollary 9. $\overline{\mathscr{Y}}_{0} \subset \mathscr{S}$ is the disjoint union of the G-orbits of the subgroups $D^{I} \in \overline{\mathscr{Y}}_{0}$. Proof. Consider a sequence $g_{n} \in G$ such that $g_{n} K g_{n}^{-1}$ converges to $D \in \mathscr{S}$. One can extract a subsequence and suppose that $g_{n}=k_{n} c_{n} a_{n} k_{n}^{\prime}$ with $k_{n}, k_{n}^{\prime} \in K$, $\lim _{n} c_{n}=c, \lim _{n} k_{n}=k$ and $a_{n}$ is I-canonical. Then from the above proposition $\lim _{n} a_{n} K a_{n}^{-1}=D^{I}$ and $\lim _{n} g_{n} K g_{n}^{-1}=k c D^{I} c^{-1} k^{-1} \in G . D^{I}$. Suppose that $g D^{I} g^{-1}=D^{J}$ for some $g, I, J$. The unipotent radical of $D^{I}$ is $N(I)$ and the normaliser of $N(I)$ is $P(I)$. It follows that $g P(I) g^{-1}=P(J)$, so that $I=J, g \in P(I)$, $D^{I}=D^{J}$.

Definition 10. For $D \in \bar{\varphi}_{0}, D=g D^{I} g^{-1}$, one defines the measure $m^{D}$ by $m^{D}=g . m_{I}$. This definition is valid because $g$ is defined up to an element of the normaliser of $D^{I}$ which is the subgroup $R^{I}$ and the stabiliser of $m_{I}$ is equal to $R^{I}$. 
Definition 11. If $i$ is the map of $X$ into the space $\mathscr{P}(\mathscr{F})$ of probability measures on $\mathscr{F}$ defined by $i(g .0)=g$. $m$, then the closure $i \overline{(X)}=\overline{G \cdot m} \subset \mathscr{P}(\mathscr{F})$ is called the maximal Satake-Furstenberg compactification of $X$.

It is shown in [Moo1] that this definition coincide with that originally given by Satake [Sa] in the context of quadratic forms. The definition given by Furstenberg [Fu 1, Moo1] is in terms of measures on $G / M A N$ and fits well to the situation considered in part II.

The meaning of $g . m$ is the present context is that of exit measure for brownian motion in $X$ starting from $g .0$.

Lemma 12. G.m is the disjoint union of the orbits of the measures $m_{I}$.

Proof. The proof is the same as that of the corollary 8 except for slight details ; if $G . m_{I} \cap G . m_{J} \neq \phi$. The stabilisers of $g . m_{I}$ and $m_{J}$ are $g R^{I} g^{-1}$ and $R^{J}$ so that $g R^{I} g^{-1}=R^{J}$ and, the same argument as, in corollary 8 with $D^{I}$ replaced by $R^{I}$ leads to $I=J, g \in R^{I}, g \cdot m_{I}=m_{I}$.

Theorem 13. The map $D \stackrel{i}{\longrightarrow} m^{D}$ is an isomorphism of $\overline{\mathscr{S}}_{0}$ onto $\overline{G . m}$.

Proof. In order to show the continuity of the map $i$, it suffices to show that if the sequence $g_{n} K_{g_{n}}^{-1}$ converges to $D$, then the sequence $g_{n} . m$ converges to $m^{D}$. If $\mu \in G . m$ is the limit of a subsequence $g_{n_{j}} . m$, one can suppose $g_{n_{j}}=k_{n_{j}} c_{n_{j}} a_{n_{j}} k_{n_{j}}^{\prime}$ with $\lim _{j} k_{n_{j}}=k, \lim _{j} c_{n_{j}}=c$ and $a_{n_{j}} I$-canonical. Then one has $D=k c D^{I} c^{-1} k^{-1}$, $\mu=k c . m_{I}$ because of lemmas $3,4,5$ and proposition 7 .

These formulae imply $\mu=m^{D}$ so that $g_{n} . m$ converges to $m^{D}$.

The density of $\mathscr{S}_{0}$ into $\overline{\mathscr{S}}_{0}$ and of $G . m$ into $\overline{G . m}$ implies that $\mu$ is surjective. In order to show that $\mu$ is injective suppose $m^{D}=m^{D^{\prime}}$, so that the relations $D=g D^{I} g^{-1}, D^{\prime}=g^{\prime} D^{J} g^{\prime-1}$ implies $g \cdot m^{I}=g^{\prime} . m^{J}$. From lemma 12, it follows $I=J, g \in g^{\prime} R^{I}$, hence $D=D^{\prime}$.

The following results give complementary informations on the distal subgroups $D^{I}$ and amenable subgroups $R^{I}$ [Zi]. The proofs remain valid for semi-simple groups over local fields and allow extensions of the results of [Moo 2].

Furthermore it gives an answer to a question of $\mathrm{H}$. Furstenberg about a possible unified conjugacy theorem for maximal compact subgroups and 
maximal amenable subgroups. Here the relevant concept is that of distal subgroups. This can be compared to [Moo 2].

Theorem 14. If $D^{I} \in \overline{\mathscr{S}}_{0}$ is contained in an amenable group $R$, then $R \subset R^{I}$. The subgroup $D^{I}$ is a maximal distal subgroup and $R^{I}$ is a maximal amenable subgroup. Every distal subgroup is contained in a maximal distal subgroup. Every amenable subgroup is contained is a maximal amenable subgroup. Every element of $\bar{Y}_{0}$ has a unique fixed point in $\bar{X} S F$.

The proof uses some lemmas of independent interest and the following proposition. A proof with the topological tools of this paper is given at the end of paragraph II. Only the first part is used here ; this follows also from proposition 4.4 and the corollaries of theorem 4.15 in [Bor-Ti1].

Proposition 15. Suppose $Q$ is a parabolic subgroup of G. Then $Q \supset N(I)$ if and only if $Q$ contains a minimal parabolic contained in $P(I)$. If $D^{I} \subset Q$, then $P(I) \subset$.

Lemma 16. Every distal subgroup is contained in a maximal one. Every amenable subgroup is contained in a maximal one.

Proof. In the two cases one used Zorn's lemma. If $D_{j}(j \in \mathscr{F})$ is a totally ordered family then $\bigcup_{j \in J} D_{j}$ is distal by definition. In the second case one uses the fixed point characterisation of amenability. If $\bigcup_{j \in J} R_{j}$ acts on a compact convex set $C$ by affine transformations, the same is true for every $R_{j}$. If $C_{j} \subset C$ is the set of fixed point of $R_{j}$, then the family $C_{j}$ has the finite intersection property, $\bigcap_{j \in J} C_{j}$ is non trivial and equal to the set of fixed point of $\cup_{j \in J} R_{j}$.

Lemma 17. Suppose that a group $H \subset G \ell(V)$ acts distally on the finite dimensional vector space $V$ and denote by $W$ the subspace of vectors $v \in V$ such that the orbit $H . v$ is relatively compact. If $v$ is an $H$-invariant measure on the projective space $P(V)$. Then $v$ is supported by the projective subspace $P(W)$.

Proof. One uses induction on $\operatorname{dim} V$, the result being trivial if $\operatorname{dim} V=1$. The result is also trivial if $W=V$. If $W \neq V$, the group $H$ is unbounded and the same contraction argument from [Fu1] used in the proof of lemma 6 leads to the fact that $v$ is supported by the union of two (strict) projective subspaces; the distality of $H$ is used through the relation $|\operatorname{det} h|=1$ for $h \in H$.

Consider now the set $\Lambda$ of finite unions of projective subspaces which support $v$. This set has a minimum element $\sigma$ because an intersection of a sequence of elements of $\Lambda$ is a finite intersection. 
The $H$-invariance of $v$ implies the $H$-invariance of $\sigma$, hence the invariance of every subspace in $\sigma$ under a subgroup $H_{0} \subset H$ of finite index. For every subspace $\sigma^{\prime} \subset \sigma$ the restriction $v^{\prime}$ of $v$ to the projective space $P\left(\sigma^{\prime}\right) \subset P(V)$ is $H_{0^{-}}$ invariant.

From the induction hypothesis and the condition $\left|H / H_{0}\right|<\infty$ one gets that $v^{\prime}$ is supported by $P(W) \cap P\left(\sigma^{\prime}\right)$. Hence $v$ is supported by $P(W)$.

Lemma 18. Consider the boundary $G / P(I)$ and the action of $N(I)$ on $G_{/ P(I)}$. The origin of $G_{/ P(I)}$ is the unique point of $G_{/ P(I)}$ which is $N(I)$-invariant.

Proof. If $x \in G / P(I)$ is $N(I)$-invariant then the stabiliser $g P(I) g^{-1}$ of $x$ is normalised by $N(I)$ : if $u \in N(I), u g P(I) g^{-1} u^{-1}=g P(I) g^{-1}$. Hence $g^{-1} u g$ normalises $P(I)$. Because the normaliser of $P(I)$ is $P(I)$ itself one has $g^{-1} N(I) g \subset P(I), N(I) \subset$ $g P(I) g^{-1}$. From the proposition 15 one gets the existence of a minimal parabolic subgroup $h P h^{-1}$ contained in $g P(I) g^{-1}[h \in P(I)]$. Hence $h^{-1} g P(I) g^{-1} h$ contains $P$ and is a standard parabolic subgroup : $h^{-1} g P(I) g^{-1} h=P(I), g^{-1} h \in P(I), g \in$ $P(I), g P(I) g^{-1}=P(I), x=\{P(I)\}$.

Lemma 19. If $\rho$ is a rationnal representation of $N(I)$ in a vector space $V$ such that the orbit of $v \in V$ under $\rho[N(I)]$ is bounded, then $v$ is invariant under $\rho[N(I)]$.

Proof. The group $N(I)$ is generated by root subgroups of $A$ in $G$ and such a root subgroup $L$ is rationnally isomorphic to the additive group $\mathbb{R}$. Hence the coordinates of $\rho(u) v(u \in L)$ in $V$ define polynomial functions on $\mathbb{R}$ which are bounded : they are constant and $\rho(u) v=v$ when $u \in L$. Because such subgroups $L$ generate $N(I)$ one has $\rho(u) v=v$ when $u \in N(I)$.

Lemma 20. The measure $m_{I}$ is the unique $D^{I}$ invariant probability measure on $\mathscr{F}$.

Proof. Consider a probability measure $v$ on $\mathscr{F}$ which is $D^{I}$-invariant. Because $B=P(\phi)$ is algebraic, there exist a rationnal representation $\rho$ of $G$ in a vector space $V$ and a vector $v \in V$ such that $\rho(B)$ is the stabiliser of the line $\bar{v} \in P(V)$ defined by $v$ [Bor]. This gives an isomorphism of $G / B$ onto the $\rho(G)$-orbit of $\bar{v} \epsilon$ $P(V)$. If $\bar{v}$ denotes the image of $v$ on $\rho(G) . \bar{v} \subset P(V)$, then $\bar{v}$ is $\rho[N(I)]$-invariant and one obtains from lemmas 17-19 that $\bar{v}$ is supported by the set of fixed points of $\rho[N(I)]$ in $\rho(G) . \bar{v}$. From lemma 18 one knows that this set of fixed points is contained in the fiber in $\mathscr{F}$ of the origin of $G / P(I)$. Hence $\bar{v}$ or $v$ are supported by this fiber. From the fact that $m_{I}$ is the unique $D^{I}$-invariant probability measure on this fiber one conclude $v=m_{I}$. 
Proof of the theorem. The action of $R$ on $\mathscr{F}$ leaves invariant a probability measure $v$ because $R$ is amenable. From lemma 20 and hypothesis $R \supset D^{I}$ one obtains $v=m_{I}$. But $R^{I}$ is the stabiliser of $m_{I}$ (lemma 7), hence $R \subset R^{I}$. If $R \supset D^{I}$ is distal, then $R \cap A$ is also distal, hence $R \cap A=\{e\}$. The relation $D^{I} \subset R \subset R^{I}=$ $A(I) D^{I}$ implies $R=D^{I}$. This prove that $D^{I}$ is a maximal distal subgroup.

The first assertion of the theorem proves the maximality of $R^{I}$. The last assertions of the theorem follows from lemmas 16 and 20 .

The following corollary uses in an essential way the properties of the real field. The results above can be extended to the local field situation.

Corollary $21 . \overline{\mathscr{S}}_{0}$ is the space of all maximal distal subgroups .

The proof uses two lemmas.

Lemma 22. Suppose that $L$ is a closed subgroup of $H \subset G \ell(V), H / L$ is compact and $L$ acts distally on $V$. Then $H$ acts distally on $V$.

Proof. One can find a compact set $C \subset H$ such that $H=C L=L C$ because the homogeneous space $H_{/ L}$ is compact. Suppose $H$ is not distal on $V$ and denote by $h_{n} \in H$ a sequence such that, for some vector $v \in V$, one has $\lim _{n} h_{n} \cdot v=0$. One can write $h_{n}=c_{n} \ell_{n}$ with $c_{n} \in C, \ell_{n} \in L$. Since $\frac{\left|\ell_{n} v\right|}{\left|h_{n} v\right|}$ is bounded by $\sup _{c \in C}\left|c^{-1}\right|<\infty$, $\lim \left|\ell_{n} v\right|=0$. This contradicts the distality of $L$.

The following appears essentially in [Ab].

Lemma 23. Every maximal distal subgroup $D$ of $G$ is a semi-direct product of a compact group $C$ and a unipotent subgroup $U$ which is normal in $D$.

Proof. It is shown in [Co-Gu] that the distality of $D$ on the Lie algebra $\varphi$ is equivalent to the existence of a $D$-invariant flag $\mathscr{S}_{1} \subset \mathscr{S}_{2} \subset \ldots \subset \mathscr{S}_{r}=\mathscr{S}$ such that $D$ acts isometrically on every quotient $\mathscr{G}_{k+1} / \mathscr{G}_{k}$. Because the property of preserving a subspace and a quadratic form are algebraic conditions, the same property are true of the Zariski-closure $Z(D)$ of $D$ in $G$. Because $D$ is distal maximal and $Z(D)$ acts distally on $\mathscr{G}$ one has $Z(D)=D$. Consequently, $D$ splits as a semi-direct product of its unipotent radical $N$ and a Levi factor $C$ which is reductive. Because of the complete reducibility of representations of $C$ and the isometry condition one obtains that $A d C$ and $C$ itself are compact. Finally $D=Z(D)=C . N$. 
Proof of the corollary. The first assertion is a direct consequence of lemma 20. In order to prove the second assertion one shows that a maximal distal subgroup is conjugate to some $D^{I}$. It is already known from theorem 14 that $D^{I}$ is maximal as a distal subgroup.

From a theorem of [Bor-Ti2] it is known that every unipotent subgroup $N$ of $G$ is contained in the unipotent radical of a parabolic subgroup $L$ such that the normaliser of $N$ is also contained in $L$. If $D$ is distal maximal the lemma above gives $D=C . N$ with $C$ compact and $N$ unipotent. The result of [Bor-Ti2] gives a parabolic subgroup $L$ with unipotent radical $V$ such that $N \subset V$ and $C . N \subset L$ because $C$ normalise $N$. Because of lemma 22, the group $C . V$ is distal. By maximality of $C . N$ one obtains $C . N=C \cdot V, N=V$. By conjugacy one can suppose $L=B(I), N=N(I), D=C . N(I)$. Because $C$ is compact and $K^{I}$ is a maximal compact subgroup of $P(I)$ there exists $\ell \in P(I)$ such that $\ell C \ell^{-1} \subset K^{I}$, and hence $\ell C N(I) \ell^{-1} \subset K^{I} N(I)=D^{I}$.

In order to give a more elementary description of $\overline{\mathscr{S}}_{0}$, denote by $\mathscr{D} \subset \mathscr{G}$ the Lie

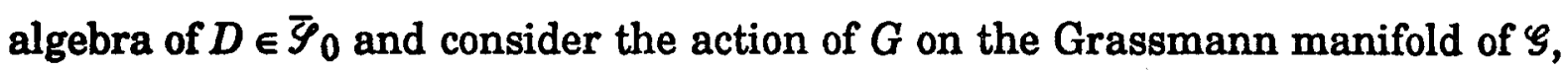
and in particular on the set of Lie subalgebras of the form $\mathscr{D}$.

Lemma 24. The map $D \rightarrow \mathscr{D}$ is injective.

Proof. One can suppose $D=D^{\prime}$ are of the form $D^{\prime}=D^{J}, D=g D^{I} g^{-1}$ and has to prove $D=D^{\prime}$ if their lie algebras $\mathscr{D}, \mathscr{D}^{\prime}$ are the equal. The unipotent radicals of $D^{J}, g D^{I} g^{-1}$ are $N(J)$ and $g N(I) g^{-1}$, hence $N(J)=g N(I) g^{-1}$. Passing to normalisers one has $P(J)=g P(I) g^{-1}$, hence $I=J, g \in P(I)$. In the same way; the stabilisers of $\mathscr{D}, \mathscr{D}^{\prime}$ in $G$ are the same $: R^{I}=g R^{I} g^{-1}, g \in R^{I}$. But then $D^{J}=D^{I}$ $=g D^{I} g^{-1}$.

Proposition 25. The map $D \rightarrow \mathscr{D}$ is a G-equivariant isomorphism of $\overline{\mathscr{Y}}_{0}$ with the closure of the G-orbit of the Lie algebra of K into the Grassmann manifold of $\hookrightarrow$.

Proof. In order to show that the map $D \rightarrow \mathscr{D}$ is continuous, consider a sequence $D_{n}$ such that $\lim _{n} D_{n}=D$ and $\lim _{n} \mathscr{D}_{n}=\mathscr{D}^{\prime}$; one has to show that $\mathscr{D}^{\prime}$ is the Lie algebra of $D$. The continuity of the exponential implies : exp $\mathscr{D}^{\prime} \subset D$. The logarithm is defined in a small neighbourhood $V$ of $e$ and the same argument leads to the fact that the Lie algebra generated by $\log (V \cap D)$ contains $\mathscr{D}^{\prime}$. It follows that $\lim _{n} \mathscr{D}_{n}=\mathscr{D}$. The map $D \rightarrow \mathscr{D}$ is injective from lemma 24 . 
Remark. From this proposition the dynamical aspects of the action of $G$ on $\bar{X} S F$ are clear ; in particular, because the action of $G$ on the Grassmann manifold is rational, the orbits are locally closed and the boundaries of the orbits are manifolds of smaller dimension than the orbits themselves [see $\mathrm{Zi}$ ].

The proximality of the action of $G$ on $\bar{X} S F$ follows from the properties of projective spaces. This property is closely related to the strong proximality of $G$ on $\mathscr{F}$ considered in [G $\ell$ ] and [Moo2].Clearly there is a unique $G$-minimal orbit, the orbit of $M N$, that is to say the $G$-space $\mathscr{F}$.

\section{II - $\lambda_{0}$-eigenfunctions and Satake-Furstenberg boundary.}

1 - Some $\lambda_{0}$-eigenfunctions and their stabilisers.

Denote by $m$ the $K$-invariant probability measure on the Furstenberg boundary $\mathscr{F}$ and recall [Fu1] that the Poisson kernel of $X$ is given by $P(g, b)=P(g .0, b)$ $=\frac{d g \cdot m}{d m}(b)=\sigma\left(g^{-1}, b\right)(g \in G, b \in \mathscr{F})$. Hence every bounded harmonic function $f$ on $X$ is given by a boundary function $\hat{f}$ on $\mathbb{L}^{\infty}(\mathscr{F})$ via the Poisson formula

$$
f(g, o)=\int P(g, b) \hat{f}(b) d m(b) .
$$

Consider the Iwasawa decomposition $G=K A N$ and write $a(g)$ for the $A$-part of $g \in G$. Denote by $2 \rho$ the sum of positive roots of the Lie algebra $a$ of $A$; hence the determinant of the restriction of Ada $(a \in A)$ to the Lie algebra of $N$ is $e^{2 p(a)}$. Then the modular function of $A N$ is given by the exponential $\delta=e^{2 \rho}$ and the Poisson kernel can be expressed [He] as : $P(g, b)=e^{-2 \rho}\left[a\left(g^{-1} k\right)\right]$ with $b \in \mathscr{F}$ corresponding to $k \in K$.

The function $\sigma$ satisfies the cocycle equation : $\sigma(g h, b)=\sigma(g, h . b) \sigma(h, b)$. Recall also $[\mathrm{Ka}, \mathrm{Gu} 2]$ that the $\lambda_{0}$-eigenfunctions of the Laplacian which are minimal are the functions $P^{1 / 2}(g, b)(b \in \mathscr{F})$ so that every positive $\lambda_{0}$-eigenfunction can be written uniquely in the form

$$
u(g . o)=\int P^{1 / 2}(g, b) d v(b)
$$

with $v$ a positive measure on $\mathscr{F}$. If $u(0)=1, v$ is a probability measure (which is the exit law in $\bar{X} S F$ for the Doob $u$-process). Denote by $h_{I}$ the $\lambda_{0}$-eigenfunction associated to $m_{I}$ by the formula

$$
h_{I}(g, o)=\int P^{1 / 2}(g, b) d m_{I}(b) .
$$

In particular $\Phi_{0}(g)=\Phi_{0}(g .0)=\int P^{1 / 2}(g, b) d m(b)$ will be called the ground state of $G$ or $G / K$. 
Consider now the set of $\lambda_{0}$-eigenfunctions $h$ normalised by the condition $h(0)=1$ and the action of $g \in G$ on this set given by

$$
S_{g} h(x)=\frac{h\left(g^{-1} \cdot x\right)}{h\left(g^{-1} \cdot 0\right)} .
$$

Consider also the twisted action of $g$ on the set of probability measures on $F$ given by

$$
S_{g}^{0} v=\frac{\int \sigma^{1 / 2}(g, b) \delta_{g . b} d v(b)}{\int \sigma^{1 / 2}(g, b) d v(b)}
$$

It is clear that if $h$ is of the form $h(x)=\int P^{1 / 2}(x, b) d v(b)$, then

$$
S_{g} h(x)=\int P^{1 / 2}(x, b) d S_{g}^{0} v(b),
$$

and the map $v \rightarrow h$ is a $G$-equivariant isomorphism. In this way, the two actions of $g$ can be identified because of uniqueness of integral representation. In probabilistic language, $v$ is the exit measure of the Doob $h$-process and $S_{g}^{0} v$ is the exit measure for the Doob $S_{g} h$-process in $\bar{X} S F$.

If $X(I)$ is the symmetric space $M(I) / K^{I}$ then the ground state $\Phi_{I}$ of $G^{I}$, or $M(I)$, $X(I)$ is given by $\Phi_{I}(g)=\Phi_{I}(g .0)=\int\left(\frac{d g \cdot m_{I}}{d m_{I}}\right)^{1 / 2}(b) d m_{I}(b)$. The functions $\Phi_{I}, h_{I}$ are related by the

Proposition 1. If $x \in X$ is written in I-horispherical coordinates : $x=u$ a s . 0 $[u \in N(I), a \in A(I), s \in M(I)]$ one has $h_{I}(x)=e^{P(a)} \Phi_{I}(s)$.

The set of group elements $g \in G$ such that $h_{I}\left(g^{-1}, x\right)$ is proportional to $h_{I}(x)$ is the subgroup $R^{I}=K^{I} A(I) N(I)$ and the coefficient of proportionality is the exponential on $R^{I}$ defined by the restriction of $e^{-\rho}$ to $A(I)$.

Before giving the proof one proves the following result

Lemma 2. The two cocycles $\sigma(g, b)=\frac{d g^{-1} \cdot m}{d m}(b), \sigma_{I}(g, b)=\frac{d g^{-1} \cdot m_{I}}{d m_{I}}(b)[g \in P(I)$, $b \in \mathscr{F}(I)]$ satisfy the equation:

$$
\sigma(g, b)=\sigma_{I}(g, b) e^{-2 p(a)}
$$

where $a$ is the diagonal part of $g$ in the Langlands decomposition of $P(I)$. 
Proof. Consider also the cocycle $\bar{\sigma}(g, b)=\frac{d g^{-1} \cdot m^{-}}{d \bar{m}}(b)$ where $\bar{m}$ denote the projection of $m$ onto $\mathrm{G} / P(I)$. The three cocycles $\sigma, \sigma_{I}, \bar{\sigma}$ on $P(I) \times \mathscr{P}(I)$ are $K^{I}$ invariant by definition. Because the measure $m$ can be identified with $m_{I} \times \bar{m}$, one has for $g \in P(I), b \in \mathscr{F}(I)$

$$
\sigma(g, b)=\sigma_{I}(g, b) \bar{\sigma}(g, b)
$$

by the $K^{I}$-invariance and equivalence (cohomology) of cocycles.

However $\bar{\sigma}(g, b)$ is defined on $G \times \mathrm{G} / P(I)$ and reduces to an exponential when $b \in P(I) / P=\mathscr{F}(I)$ and $g \in P(I)$. This exponential is trivial on the group $M(I)=G^{I} M$ and needs only to be calculated on $R^{I}$ because $P(I)=M(I) R^{I}$. One uses again the relation between the three cocycles $\sigma, \sigma_{I}, \bar{\sigma}$. For $g=s a u[s \in M(I), a \in A(I), u \in$ $N(I)]$ and $b=k B\left(k \in K^{I}\right)$ one has

$g k=s k a\left(k^{-1} u k\right)$ with $k^{-1} u k \in N(I)$ because $K^{I}$ normalises $N(I)$ and centralises $A(I)$.

It follows that and if

$$
g \cdot b=s \cdot b
$$

then

$$
\begin{aligned}
& g=r=s \in R^{I} \\
& a(r k)=a(r)=a \text { and } r . m_{I}=m_{I} .
\end{aligned}
$$

If $g \in A(I) N(I)$, then $g . b=b$.

Hence $\sigma(r, b)=e^{-2 \rho[a(r k)]}=e^{-2 \rho(a) \text {. }}$

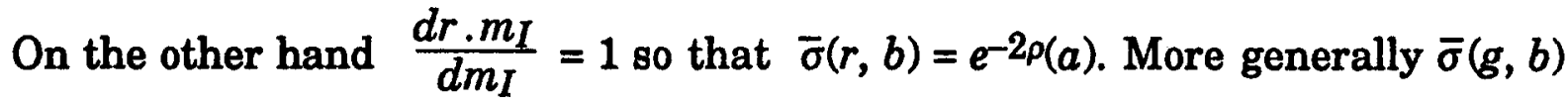
$=e^{-2 \rho(a)}$ for $g \in P(I)$ because the two sides are exponential on $P(I)$, trivial on $M(\Lambda)$, and coincide on $R^{I}$. Using again the relation between $\sigma, \bar{\sigma}, \sigma_{I}$, one obtains $\sigma(g, b)=\sigma_{I}(g, b) e^{-2 \rho(a)}$.

Proof of the proposition. Write $x=u$ as .0 and :

$h_{I}(x)=\int \sigma^{1 / 2}\left(s^{-1} a^{-1} u^{-1}, b\right) d m_{I}(b)=e \rho(a) \int \sigma_{I}^{1 / 2}\left(s^{-1} a^{-1} u^{-1}, b\right) d m_{I}(b)$. Because $\sigma_{I}(r, b)=1$ if $r \in R^{I}$, and $a^{-1} u^{-1}, b=b$ for $b \in \mathscr{F}(I)$ one has $\sigma^{1 / 2}\left(s^{-1} a^{-1} u^{-1}, b\right)=$ $\sigma^{1 / 2}\left(s^{-1}, b\right)$ and $h_{I}(x)=e^{\rho(a)} \Phi_{I}(s, 0)$.

Observe that, in the Langlands decomposition of $g \in P(I)$, the $A(I)$-component and the $M(I)$-component define homomorphisms of $P(I)$ onto $A(I)$ and $M(I)$. If $x=g . o=u a s .0$, and $r \in R^{I}$, the $A(I)$ component of $r^{-1} g$ is $a\left(r^{-1}\right)$ and the $M(I)$ component is multiplied by an element $k$ of $K^{I}$, so that $\Phi_{I}\left(k^{-1} s .0\right)=\Phi_{I}(s .0)$. 
Finally $h_{I}\left(r^{-1}, x\right)=e^{-\rho}[a(r)] h_{I}(x)$ where $r \in R^{I}$. Conversely denote by $L$ the subgroup of elements $g \in G$ such that $h_{I}\left(g^{-1} \cdot x\right)$ is proportionnal to $h_{I}(x)$, that is to say

$$
h_{I}\left(g^{-1} x\right)=c(g) h_{I}(x)
$$

with some exponential $c(g)$ on $L$. By definition of $h_{I}$ and the above calculation one has $L \supset R^{I}$. For $g \in L$ one has $S_{g} h_{I}=h_{I}$, so that $S_{g}^{0} m_{I}=m_{I}$. It follows that the supports of these two measures are equal that is to say $g P\left(I_{/ B}=P(I)_{/ B}\right.$ $(g \in P(I))$. So that $R^{I} \subset L \subset P(I)$. The equation $S_{g}^{0} m_{I}=m_{I}$ can be written for $g \in M(I)$ in the form

$$
\sigma_{I}^{-1 / 2}\left(g^{-1}, .\right) g \cdot m_{I}=c(g) m_{I}=\sigma_{I}^{1 / 2}\left(g^{-1}, .\right) m_{I} .
$$

Hence if $g \in M(I) \cap L$, one has $\sigma_{I}^{1 / 2}\left(g^{-1},.\right)=c(g), g . m_{I}=m_{I}, c(g)=1$.

The second condition and lemma 6 of the paragraph I gives : $g \in K^{I}$. Hence $L=(M(I) \cap L) R^{I}=R^{I}$ and $c(r)=e^{-\rho}[a(r)]$.

Definition 3. For $D^{I}=K^{I} N(I)$ and $D=g D^{I} g^{-1} \in \overline{\mathscr{S}}_{0}$ one denotes by $h^{D}$ the $\lambda_{0^{-}}$ eigen function defined by

$$
h^{D}(x)=S_{g} h_{l}(x) .
$$

Remark. This definition is valid because the formula $D=g D^{I} g^{-1}$ defines the element $g$ up to an element of the normaliser $R^{I}$ of $D^{I}$ and from the above proposition $S_{r} h_{I}=h_{I}$ for $r \in R^{I}$. The function $h^{D}$ has the following integral representation : $h^{D}(x)=\int P^{1 / 2}(x, b) d S_{g}^{0} m_{I}$. The measure $m_{*}^{D}=S_{g}^{0} m_{I}$ is equivalent to the measure $g . m_{I}$ and its support is equal to the fiber $g . \mathscr{F}(I)$ in $\mathscr{F}$ of the point $g . P(I) \in G / P(I)$. From the above definitions and the proposition one has the following :

Corollary 4. The stabiliser of the measure $m_{*}^{D}$ in the twisted action $S_{g}^{0}(g \in G)$ is the normaliser $R(D)$ of $D$. The set of elements $g \in G$ such that $h^{D}\left(g^{-1} \cdot x\right)$ is proportionnal to $h^{D}(x)$ is the subgroup $R(D)$ and the coefficient of proportionnality is the square root of the modular function of $R(D)$.

\section{2) Ground state properties.}

The following will be fundamental in what follows 
Theorem 5. For $D \in \overline{\mathscr{S}}_{0}$, the function $h^{D}$ is the unique positive normalised $\lambda_{0}-$ eigen function which is invariant under left translations by $D$.

It is clear, by definition of $h^{D}$, that it suffices to prove the theorem when $D=D^{I}$. The proof will follow from some propositions of a more general character which involve positive measures on a locally compact group $H$.

These results will be used in the cases $H=G$ or $H=M(I), H=P(I)$.

Definition 6. If $p$ is a positive and bounded measure on the locally compact group $H$, one says that $p$ is well behaved if $p$ has a continuous density with compact support $S$ and $H=\bar{\cup} \overline{S^{n}}$.

Remark. If $p$ is well behaved, then $H$ is compactly generated by $S$.

If $p$ is well behaved and $K$-bi-invariant on the semi-simple group $G$, a direct analogue of $\lambda_{0}$ is given by

$$
r_{0}=\int \Phi_{0}(g) d p(g)
$$

because the properties of spherical functions [He] and $K$-biinvariance of $p$ implies : $r_{0} \Phi_{0}=\Phi_{0} * p$.

The theorem 5 will be a consequence of the

Theorem 5'. Suppose $p$ is well behaved and $K$-bi-invariant. For $D \in \overline{\mathscr{S}}_{0}$, the function $h^{D}$ is the unique normalised $r_{0}$-eigen function of the right convolution by $p$ which is invariant under left translation by $D$.

The plan of the proof is to use the decompositions $G=P(I) K, P(I)=N(I) A(I)$ $M(I)$, the properties of the group $Z=A(I) M(I)$, and to reduce the equations to equations on $Z, M(I), G^{I}$ where uniqueness properties of the ground state are available. One has to study properties of $Z$ and of the decomposition $G=P(I) K$ in a more general context of locally compact groups.

One considers first a semi-simple group $G$, a maximal compact subgroup $K$ and the Iwasawa decomposition $G=K A N$. For every exponential $\ell \in A^{*}$, the spherical function $\Phi^{\ell}$ is defined by $\Phi^{\ell}(g)=\int \ell\left[a\left(g^{-1} k\right)\right] d m(k)$ where $m$ is the Haar measure on $K$. If $p$ is a well-behaved $K$-bi-invariant probability measure on $G$ one has $\Phi^{\ell} * p=\hat{p}(\ell) \Phi^{\ell}$ for some positive number $\hat{p}(\ell)$ [He]. The function $\hat{p}(\ell)$ on $A^{*}$ is called the Laplace transform of $p$. In particular, if $\ell=e^{p}$, then $p^{\gamma}(\ell)$ $=r_{0}$ and $\Phi^{\ell}=\Phi_{0}$. With these notations one has the 
Proposition 7. Suppose $G$ is semi-simple and $p$ is well behaved and $K$-biinvariant. Then the spectral radius of the convolution operator on $\mathbb{L}^{2}(G)$ defined by $p$ is equal to $r_{0}=\operatorname{Inf}_{\ell \in A^{*}} \hat{p}(\ell)$.

Proof. The formula is clear from the above discussion because

$$
\Phi_{0} \leq \Phi^{\ell} \quad \forall \ell \in A^{*} .
$$

Consider the regular representation $\gamma$ of $G$ into $\mathbb{L}^{2}(G / B)$ :

$$
\gamma(g)[\varphi](x)=\left(\frac{d g m}{d m}\right)^{1 / 2}(x) \varphi\left(g^{-1} x\right)
$$

where $\varphi \in \mathbb{L}^{2}(G / B), x \in G / B$.

The functions $h_{x}(g)$ defined by $h_{x}(g)=\left(\frac{d g m}{d m}\right)^{1 / 2}(x)$ are eigenfunctions of the right convolution by the $K$-bi-invariant measure $p$ :

$$
\int\left(\frac{d g h^{-1}}{d m}\right)^{1 / 2}(x) d p(h)=C\left(\frac{d g m}{d m}\right)^{1 / 2}(x)
$$

with a constant $C$ independent of $x$.

By integration with respect to $m$ :

$$
\begin{aligned}
& \Phi_{0}(g)=\int\left(\frac{d g m}{d m}\right)^{1 / 2}(x) d m(x) \\
& \Phi_{0} * p=C \Phi_{0} \\
& r_{0}=C .
\end{aligned}
$$

Also, taking $g=e, \gamma(p) 1(x)=C 1(x)$.

On the other hand $\gamma(p)$ is a positive and compact operator on $\mathbb{L}^{2}\left(G_{/ B}\right)$ : its dominant eigenvalue is $r_{0}$ and is equal to its spectral radius. Hence $r_{0}$ is the spectral radius of $\gamma(p)$ in $\mathbb{L}^{2}(G / B)$.

It is well known that the spectral radius of a positive convolution operator increases when the regular representation in $\mathbb{L}^{2}(G)$ is replaced by the regular representation in $\mathbb{L}^{2}(G / H)$, for any closed subgroup $H$. Here $H=B=M A N$ is amenable [Zi], hence the identity representation of $B$ is weakly contained in the regular representation in $\mathbb{L}^{2}(B)$. By the continuity of the inducing process from $B$ to $G$, one obtains that the regular representation of $G$ in $\mathbb{L}^{2}(G / B)$ is weakly contained in the regular representation in $\mathbb{L}^{2}(G)$. Hence the spectral radius of $\gamma(p)$ is dominated by the spectral radius of the convolution in $\mathbb{L}^{2}(G)$. Finally $r_{0}$ is equal to this last spectral radius. 
Definition 8. Suppose $H$ is locally compact, $p$ is a well behaved positive and bounded measure on $H$ and $\Lambda$ is a closed subgroup. One denotes by $r(\Lambda, p)$ the infimum of numbers $C>0$ such that there exist some left $\Lambda$-invariant Radon measure $\mu$ such that

$$
\mu * p=C \mu \text {. }
$$

If $\Lambda=e$, one denote $r(\Lambda, p)=r(p)$.

\section{Remarks.}

a) The set of measures $\mu$ considered above is non trivial : it contains the leftinvariant Haar measure if $C$ Is equal to the total mass of $p$.

It is easy to show from compactness arguments that the infimum $r(\Lambda, p)$ is attained.

b) The number $r(p)$ has an important probabilistic significance for the random walk generated by $p$. It is shown in [Gu1] that, if $p$ is well behaved, one has $r(p)=\lim _{n} p^{n}(e)^{1 / n} ;$ hence $r^{-1}(p)$ is the radius of convergence (in the weak topology) of the resolvent $(I-z p)^{-1}=\sum_{0}^{\infty} z^{n} p^{n}$.

Definition 9. The pair $(\Lambda, p)$ is said to satisfy the ground state property if the system of convolution equations

$$
\begin{aligned}
& r(\Lambda, p) p=\mu * p \\
& \forall h \in \Lambda: \delta_{h} * \mu=\mu
\end{aligned}
$$

has a unique normalised solution.

\section{Remarks.}

a) Because $p$ is well behaved the measure $\mu$ has a continuous density $f$ with respect to right invariant Haar measure and satisfies $f * p=r(\Lambda, p) f$. The normalisation is $f(e)=1$, the corresponding measure is denoted $\varphi_{\Lambda_{x} p}$ and identified below with its density.

b) It is easy to show that if $H=\mathbb{R}^{d}$, and $p$ well behaved every pair $(\Lambda, p)$ has the ground state property. If $\hat{p}(\ell)\left(\ell \in H^{*}\right)$ denotes the Laplace transform of $p$, then $\varphi_{\Lambda, p}$ is the unique exponential $\ell$ such that $\hat{p}(\ell)=r(\Lambda, p)$. Uniqueness of $\ell$ follows from the strict convexity of $\log \hat{p}(\ell)$.

One can now give a complement to proposition 7 . 
Proposition 7'. Suppose $G$ is semi-simple, $K$ is a maximal compact subgroup and $p$ is $K$-bi-invariant. Then the pair $(K, p)$ has the ground state property. Moreover $r(p)=r(K, p)=r_{0}=\lim _{n} p^{n}(e)^{1 / n}, \varphi_{K, p}=\Phi_{0}$.

Proof. It is proved in [Gu1] that $r(p)=\lim _{n} p^{n}(e)^{1 / n}$. The relation $r(p)=r(K, p)$ follows from the definitions because $K$ is compact. The definition of $r_{0}$ gives $r(K, p) \leq r_{0}$. It is shown in [Fu2], that the relation $\mu * p=c \mu$ implies the existence of a spherical function $\Phi^{\ell}$ such that $\Phi^{\ell} * p=c \Phi^{\ell}$, hence $c=\hat{p}(\ell)$, $r(K, p) \geq \operatorname{Inf}_{\ell} \hat{p}(\ell)$. From proposition 7 one gets $r_{0} \leq r(K, p)$, hence $r_{0}=r(K, p)$.

One considers now the behaviour of the ground state property with respect to the processes of restriction to subgroups and factor groups.

Proposition 10. Suppose $H$ is a semi-direct product of the normal subgroup $V$ by the subgroup $Z$ and $\Lambda \supset V$. Denote by $\bar{p}$ the projection of $p$ on $Z$, by $\eta$ the left Haar measure on Vand write $\bar{\Lambda}=\Lambda \cap Z$. Then $r(\Lambda, p)=r(\bar{\Lambda}, \bar{p})$. If $(\bar{\Lambda}, \bar{p})$ has the ground state property, the same is valid for $(\Lambda, p)$ and $\varphi \wedge, p=\eta * \varphi_{\Lambda_{\bar{p}} \bar{p}}$.

Proof. Suppose $\mu$ is a Radon measure on $G$ which is $V$-invariant. Then it can be uniquely written

$$
\mu=\eta * \bar{\mu}
$$

with $\bar{\mu}$ a Radon measure on $Z$. Furthermore $\Lambda$-invariance of $\mu$ is equivalent to $\pi$-invariance of $\bar{\mu}$ because $\Lambda=V$. $\pi$. An easy calculation gives $\eta * \bar{\mu} * p=\eta * \bar{\mu} * \bar{p}$.

From these relations it follows that the system :

$$
\begin{aligned}
& \mu * p=c \mu \\
& \delta_{h} * \mu=\mu \quad(h \in \Lambda)
\end{aligned}
$$

is equivalent to the system

$$
\begin{aligned}
& \bar{\mu} * \bar{p}=c \bar{\mu} \\
& \delta_{h} * \bar{\mu}=\bar{\mu} \quad(h \in \bar{X}) .
\end{aligned}
$$

Hence $r(\Lambda, p)=r(\pi, \bar{p})$ by definition of $r$.

If $c=r(\pi, \bar{p}), \bar{\mu}$ is unique from the ground state property and the solution $\mu$ of

$$
\left\{\begin{array}{l}
\delta_{h} * \mu=\mu \\
\mu * p=c \mu
\end{array} \quad(h \in \Lambda)\right.
$$


is uniquely defined by

$$
\mu=\eta * \bar{\mu} .
$$

One considers now a compact subgroup $K$ of $H$ and a closed subgroup $B$ of $H$ such that the product $B K$ is equal to $H$. One denotes $K^{\prime}=B \cap K, m, m^{\prime}$ the Haar measures on $K$ and $K^{\prime}$.

One defines a kernel $\pi$ from $H$ to $B$ by the formula

$$
\pi(g, X)=\delta_{b} * m^{\prime}(X)=m^{\prime}\left(b^{-1} X\right)
$$

if $g=b k(b \in B, k \in K)$ and $X \subset B$.

The definition of $\pi$ takes care of the fact that the decomposition $g=b k$ is not unique. It follows from the lemma below that $\pi$ is a homomorphism of the algebra of $K$-bi-invariant measure in $H$ into the algebra of $K^{\prime}$-bi-invariant measure in $B$. In particular, commutativity is preserved by $\pi$. Moreover if $b \in B, k \in K$, one has by definition $\pi\left(\delta_{b} * v\right)=\delta_{b} * \pi(v), \pi\left(v * \delta_{k}\right)=\pi(v)$. Furthermore if $v=v * m$, then $v=\pi(v) * m$.

Lemma 11. Suppose $\mu, v$ are measures on $H$ and $v$ satisfies $m * v=v$. Then $\pi(\mu * v)$ $=\pi(\mu) * \pi(v)$.

Proof. Because $\pi$ is linear it suffices to check the case $\mu=\delta_{g}, v=m * \delta_{h}(g, h \in H)$. Then if $g=b k(b \in B, k \in K)$ one has

$$
\begin{aligned}
& \pi(\mu * v)=\pi\left(\delta_{b} * \delta_{k} * m * \delta_{h}\right) \\
= & \pi\left(\delta_{b} * m * \delta_{h}\right)=\delta_{b} * \pi\left(m * \delta_{n}\right)=\delta_{b} * \pi(v) \\
= & \delta_{b} * m^{\prime} * \pi(v)=\pi(\mu) * \pi(v)
\end{aligned}
$$

because $m^{\prime} * \pi(v)=\pi\left(m^{\prime} * v\right)=\pi(v)$.

As a simple corollary one has the

Lemma 12. With the notations of the above lemma, suppose that $p$ is a $K$-biinvariant probability measure on $H, \mu$ satisfies $\mu * p=\mu$. Then $\pi(\mu) * \pi(p)=\pi(\mu)$. Moreover $\pi(\mu)$ is $K^{\prime}$-bi-invariant. If $\mu$ is left-invariant under a subgroup of $B$, the same is true of $\pi(\mu)$.

With these notations one has the 
Proposition 13. Suppose $H$ is of the form $H=B K$ with $K a$ compact subgroup and $B$ a closed subgroup. Suppose $p$ is a $K$-invariant probability measure and $D$ is a closed subgroup of $B$.

Then one has $r(D, p)=r[D, \pi(p)]$.

Furthermore the ground state property for $(D, p)$ and $[D, \pi(p)]$ are equivalent. Moreover

$$
\pi\left[\varphi_{D, p}\right]=\varphi_{D, \pi(p)}=\varphi_{D, p}=\varphi_{D, \pi(p)} * m .
$$

Proof. One observes that if $\mu * m=\mu$, then $\mu=\pi(\mu) * m$. Suppose $\mu$ satisfies

$$
\begin{aligned}
& \mu * p=c \mu \\
& \delta_{d} * \mu=\mu \quad(d \in D) .
\end{aligned}
$$

Then the condition $m * p=p$ and the above lemma imply

$$
\begin{aligned}
& \pi(\mu) * \pi(p)=c \pi(\mu) \\
& \delta_{d} * \pi(\mu)=\pi(\mu) .
\end{aligned}
$$

Conversely if on $B$ one has :

$$
\begin{aligned}
& v * \pi(p)=c v \\
& \delta_{d} * v=v \quad
\end{aligned} \quad(d \in D)
$$

it follows :

$$
\begin{aligned}
& v * \pi(p) * m=c v * m \\
& \delta_{d} *(v * m)=v * m .
\end{aligned}
$$

The first equation can be written

$$
v * p=c v * m
$$

because $p=p * m=\pi(p) * m$.

From $p=m * p$, if follows

$$
v * p=(v * m) * p=c(v * m) .
$$

Hence $r(D, p)=r[D, \pi(p)]$. The last statement follows from these calculations.

Definition 14. Suppose $K$ is a compact subgroup of the locally compact group $S$. Then $K$ is said to satisfy the strong ground state property if for every $K$-biinvariant well behaved probability measure $p$, the pair $(K, p)$ has the ground state property and furthermore the measure $\varphi_{K, p}$ is independent of $p$. In this case one denotes $\varphi_{K}=\varphi_{K, p}$ and call $\varphi_{K}$ the $K$-ground state of $S$. 
Remark. If $G$ is a semi-simple real Lie group it follows from proposition 7 ' that any maximal compact subgroup has the strong ground state property. The same is true for groups of Euclidean motions.

Proposition 15. Suppose $S, A$ are closed subgroups of the locally compact group $H$ such that $H=S \cdot A, A$ is central, compactly generated in $H$ and $S \cap A$ is compact. Suppose $K \subset S$ has the strong ground state property relative to $S$. Then, if $p$ denotes a well behaved $K$-bi-invariant probability measure on $H$, the pair $(K, p)$ has the ground state property. Furthermore there exist an exponential $\ell$ on $A$ such that $\varphi_{K, p}=$ $\varphi_{K} * \ell$.

\section{Proof.}

a) One considers a minimal solution $\mu$ of the equation $\mu * p=c \mu$. Because $A$ is central in $G$ one has $c \delta_{a} * \mu=\mu * \delta_{a} * p$.

Because $p$ is well behaved, there exist constants $\Delta(a)>0, k(a) \in N$ such that $\delta_{a} * p \leq \Delta(a) p^{k(a)}$. Hence $\mu * \delta_{a} * p \leq \Delta(a) \mu * p^{k(a)}=\Delta(a) c^{k(a)} \mu$.

Hence $\delta_{a} * \mu \leq \Delta(a) c^{k(a)-1} \mu$.

The minimality of $\mu$ and the fact that $\delta_{a} * \mu$ is also a solution imply that, for some constant $\ell(a)$

$$
\delta_{a} * \mu=\ell(a) \mu .
$$

Clearly $\ell(a)$ is an exponential on $A$. Because $S \cap A$ is compact one has $\ell(S \cap A)=\{1\}$, hence one can extend $\ell$ to $G$ from the formula

$$
\ell(s a)=\ell(a) \quad(s \in S, a \in A) .
$$

It follows that there exist a positive function $\Psi$ on $S$ such that the density of $\mu$ is the product $\Psi . \ell$. One can now reduce the equation $\mu * p=c \mu$ to $S$. If $p^{\ell}$ denotes the projection of $p \cdot \ell^{-1}$ on $S$ one has $\Psi * p^{\ell}=c \Psi$.

b) If $c=r(K, p)$, it follows from the above observation that

$$
r(K, p) \leq r(K, p) \text {. }
$$

Conversely if $\varphi$ is $K$-invariant and satisfies $\varphi * p^{\ell}=r_{(K, p l)} \varphi$ one obtains $f * p=$ $r_{\left(K, p^{\ell}\right)} f$ with $f=\varphi$. $\ell$. Because $f$ is $K$-invariant one has $r(K, p) \leq r\left(K, p^{\ell}\right)$, hence $r(K, p)=r\left(K, p^{\ell}\right)$ for every exponential $\ell$ defined as above.

c) One shows now that $\ell$ is unique.

Suppose $f, f^{\prime}$ are minimal solutions of the equation

$$
\mu * p=r\left(K_{*} p\right) \mu \text {. }
$$


It is convenient here to consider $f, f^{\prime}$ as positive functions on $G$ with $f(e)=1=$ $f^{\prime}(e) \quad f=\Psi . \ell \quad f^{\prime}=\Psi^{\prime} . \ell^{\prime}$ where $\Psi, \Psi^{\prime}$ are $K$-invariant functions on $S$. From b) one has

$$
\begin{aligned}
& \Psi * p^{\ell}=r(K, p) \Psi=r\left(K, p^{\ell}\right) \Psi \\
& \Psi^{\prime} * p^{\ell^{\prime}}=r(K, p) \Psi^{\prime}=r\left(K, p^{\ell^{\prime}}\right) \Psi^{\prime} .
\end{aligned}
$$

The strong ground state property implies $\Psi=\Psi^{\prime}=\varphi_{K}$.

Consider positive numbers $\alpha, \alpha^{\prime}$ with $\alpha+\alpha^{\prime}=1$ and the function

$$
g=f^{\alpha} f^{\prime \alpha^{\prime}}=\varphi_{K} \ell^{\alpha} \ell^{\prime \alpha^{\prime}}=\varphi_{K} \lambda .
$$

From Hölder inequality one gets

$$
g * p \leq r\left(K_{2} p\right) g
$$

with equality if and only if $f=f^{\prime}$ because $p$ is well behaved. On the other hand, the strong ground state property implies

$$
\begin{aligned}
& \varphi_{K} * p^{\lambda}=r\left(K, p^{\lambda}\right) \varphi_{K} \\
& g * p=r\left(K_{2} p^{\lambda}\right) g .
\end{aligned}
$$

Hence $\left.r_{(K, p)} \geq r_{K, p}\right)$.

The relation $g * p \leq r\left(K_{2} p\right) g$ gives

$$
\begin{aligned}
& r\left(K, p^{\lambda}\right) \leq r(K, p), \text { hence } \\
& r\left(K, p^{\lambda}\right)=r(K, p), g * p=r(K, p) g .
\end{aligned}
$$

The fact that one has equality in the Hölder inequality gives $f=f^{\prime} \quad \ell=\ell^{\prime}$. Hence $\ell$ is unique. If $f$ is a normalised minimal solution of the system $f * p=$ $r\left(K_{2} p\right) f \quad \delta_{k} * f=f$, one conclude

$$
f=\varphi_{K} \cdot \ell .
$$

The measure $\mu$ corresponding to $f$ can also be written as $\mu=\varphi_{K} * \ell$ if $\ell$ is considered as a measure on $A$.

Proposition 16. Suppose $L, S$ and $M$ are closed subgroups of $H, H=S . M, L \subset S$, and moreover $L$. $M$ is a compact subgroup of $H$. If $L$ has the strong ground state property in $S$, the same is true of L. M in $H$. 
Proof. This is a simple application of proposition 13. Il $p$ is well behaved and $L . M$ bi-invariant, the measure $\pi(p)$ defined on $S$ will be $L . M \cap S$ bi-invariant, hence $L$-bi-invariant. If one denotes by $\rho$ the Haar-measure on $M$ and $\varphi_{L}$ the $L$ ground state on $S$, the $L . M$ ground state of $S . M=H$ is $\varphi L * \rho$.

\section{3) A correspondance between some $\lambda_{0}$-eigenfunctions and distal subgroups.}

In order to prove theorems $5,5^{\prime}$ which give this correspondance one return to the notations of paragraph 1 and prepare the

Proposition 17. Suppose $K$ is a maximal compact subgroup of $G$ which contains $M$. Then the subgroup $K^{I}=K \cap M(I)$ of $M(I)$ has the strong ground state property. If $p$ is a $K$-bi-invariant and well behaved probability measure on $G$, the pair $\left(D^{I}, p\right)$ has theground stateproperty.

Proof. One has to collect the informations given in paragraph 2. It has been shown in the recollections that $K_{0}^{I}=G^{I} \cap K$ is a maximal compact subgroup of the semi-simple group $G^{I}$. Clearly $M(I)=G^{I} M$ and $K_{0}^{I} M=K^{I}=K \cap M(I)$ is a group. The strong ground state property is true for $K_{0}^{I} \subset G^{I}$, because of proposition 7', hence the same is true for $K^{I} \subset M(I)$ because of proposition 16 . Because $P(I) \supset B$ and $G=B . K$ one has $G=P(I) . K$; one denotes $\pi$ the natural kernel from $G$ to $P(I)$ considered in proposition 13. One has the Langlands decomposition $P(I)=M(I) A(I) N(I)$ and the relation $D^{I}=K^{I} N(I) \subset P(I)$. Because of proposition 13, if suffice to show that $\left[D^{I}, \pi(p)\right]$ has the ground state property. Because of proposition 10 and of relation $D^{I} \supset N(I)$, the problem reduces to $M(I) A(I)$ with $\bar{p}$ and $K^{I}=D^{I} \cap M(I) A(I)$ instead of $\pi(p)$ and $D^{I}$. Because of proposition 15 and the facts that $A(I) \subset M(I) A(I)$ is central and $M(I)$ has the strong ground state property, one obtains the validity of the ground state property for $\left(K^{I}, \bar{p}\right)$.

Proof of theorem 5'. It suffice to show that $r_{0}=r\left(D^{I}, p\right)$ because of proposition 17. From proposition 7 , $r_{0} \leq r\left(D^{I}, p\right)$. From paragraph 1 , the fonction $h_{I}$ is $D^{I}$ invariant and satisfies $h_{I} * p=r_{0} h_{I}$, hence $r_{0} \geq r\left(D^{I}, p\right), r_{0}=r\left(D^{I}, p\right)$.

The following result allows one to reduce the Laplace equation to an integral equation.

Lemma 18. Suppose $\lambda \leq \lambda_{0}$. There exists a continuous and positive function $p^{\lambda}$ with compact support such that $p^{\lambda(0)}>0, p^{\lambda}(k, x)=p^{\lambda}(x)$ for every $x \in X$ and $k \in K$ with the following property : every function $f$ which satisfies $L f+\lambda f=0$ satisfies also $\forall g \in G: f(g .0)=\int f(g . x) d p^{\lambda}(x)$. 
Proof. Consider a (positive) spherical function $\Phi$ such that $L \Phi+\lambda \Phi=0$ and the differential operator on $X$ defined by

$$
D \varphi=\frac{1}{\Phi} L(\varphi \Phi)+\lambda \varphi .
$$

Then $D 1=0$ and the heat kernel associated with $D$ is given by $e^{-\lambda t} \frac{1}{\Phi(x)} p^{t(x, y) \Phi(y)}$ where $p^{t}(x, y)$ is the heat kernel associated with $L$. Denote by $q_{r}^{\lambda}$ the hitting measure of the sphere of radius $r$ centered at 0 for the brownian motion associated with $D$ and starting from 0 . Then $L f+\lambda f=0$ implies $D \frac{f}{\Phi}=0$ and $\frac{f}{\Phi}$ $(0)=\int \frac{f}{\Phi}(x) d q_{r}^{\lambda}(x)$

$$
f(0)=\int f(x) \frac{d q_{r}^{\lambda}(x)}{\Phi(x)}
$$

Now one modifies $q_{r}^{\lambda}$ as follows.

The measure $q$ defined by integration in $r$ on an interval $(1 \leq r \leq 2)$ of the individual measures $\frac{d q_{r}^{\lambda}}{\Phi}$ has an $\mathbb{L}^{\infty}$ density $q^{\lambda}(x)$ with support in the shell $1 \leq r \leq 2$ and is $K$-invariant by definition.

Furthermore, $f(0)=\int f(x) q^{\lambda}(x) d x$.

Because $f(g \cdot x)$ is also a $\lambda$-eigenfunction one has

$$
f(g .0)=\int f(g \cdot x) q^{\lambda}(x) d x .
$$

The functions $q^{\lambda}$ and $f$ on $X$ can also be considered as functions on $G$ and then the equation above reads

$$
f=f * q^{\lambda}
$$

where $q^{\lambda}$ is $K$-bi-invariant and $f$ is right $K$-invariant. The convolution $p^{\lambda}=q^{\lambda} *$ $q^{\lambda}$ defines a $K$-bi-invariant continuous function $p^{\lambda}$ with compact support and $p^{\lambda}(e)>0$. It defines on $X$ a $K$-invariant function as in the lemma.

Proof of the theorem 5. Observe that $\Phi_{0}(g)=\int P^{1 / 2}(g .0, b) d m(b)$ satisifes $L \Phi_{0}+$ $\lambda_{0} \Phi_{0}=0$. One has also $L h^{D}+\lambda_{0} h^{D}=0 L h+\lambda_{0} h=0$, one knows that $h$ and $h^{D}$ are left-D-invariant and has to show that $h$ is proportionnal to $h^{D}$. From lemma 18 one obtains a $K$-bi-invariant measure $p^{\lambda_{0}}=p$ on $G$ which is well behaved and satisfies

$$
\Phi_{0} * p=\Phi_{0}, h^{D} * p=h^{D}, h * p=h
$$


The first equation and the definition of $r_{0}$ implies $r_{0}=1$. The theorem $5^{\prime}$ implies that $h$ is proportionnal to $h^{D}$.

Corollary 19. In the topology of $\overline{\mathscr{S}}_{0}$ :

$$
\lim _{y \rightarrow D} \frac{G_{\lambda_{0}}(x, y)}{G_{\lambda_{0}}(0, y)}=h^{D}(x)
$$

uniformly on compact subsets of $X$.

Proof. Any limit function $h$ along a subsequence $y_{n}$ is a uniform limit of normalised functions invariant under the subgroups $y_{n} K y_{n}^{-1}$. Hence $h$ is $D$ invariant by the definition of the topology of $\overline{\mathscr{S}}_{0}$. From the theorem one gets $h=h^{D}$ and the corollary is proved.

Theorem 20. The Satake-Furstenberg compactification $\bar{X} S F=\overline{\mathscr{S}}_{0}$ is isomorphic to the Martin compactification of $\mathrm{X}$ at $\lambda_{0}$. At the boundary the isomorphism is given by $D \rightarrow h^{D}$.

Proof. Because of corollary 19 it suffices to check that the equality $h^{D}=h^{D^{\prime}}$ implies $D=D^{\prime}$. Since the equality of stabilisers, corollary 4 implies $R(D)=R\left(D^{\prime}\right)$. From the formula $R^{I}=K^{I} A(I) N(I)$, one sees that the exponentials of $R(D)$ are trivial exactly on the normal subgroup $D$ so that $D=D^{\prime}$.

One returns now to the random walk situation and consider the potential kernel

$$
V_{r}=\sum_{0}^{\infty} r^{-n} p^{n}
$$

The series converges in the weak topology of measures for

$$
r \geq \lim _{n} p^{n}(e)^{1 / n}=r_{0}
$$

The convergence at $r_{0}$ follows from the non amenability of $G$ [Gu1].

The equation

$$
V_{r} * p=p * V_{r}=r\left(V_{r}-\delta_{e}\right)
$$

and the fact that $p$ is well behaved shows that $V_{r}$ has a continuous density outside $e$, again denoted by $V_{r}$. The corresponding left-invariant convolution kernel is also denote by $V_{r}$ :

$$
V_{r}(x, y)=V_{r}\left(y^{-1} x\right) \quad(x \neq y) .
$$

This kernel can be considered as a kernel on $G_{/ K}$ if $p$ is $K$-bi-invariant. For each fixed $y \quad V_{r}(x, y)$ defines an $r$-eigenfunction on the domain $X / y$.

The equation above implies easily the following because $p$ is well behaved. 
Lemma 21. Suppose $C$ is a compact neighbourhood of the identity in $G$ and $R \geq r_{0}$ is a given number. Then the family of functions

$$
K^{r}(x, y)=\frac{V_{r}(x, y)}{V_{r}(e, y)}
$$

is uniformly equicontinuous on $C$ when $y \notin C^{2}$ and $r_{0} \leq r \leq R$.

This lemma implies that the Martin compactification at $r \geq r_{0}$, relative to $p$ can be defined as above ; the points of the Martin boundary define normalised $r$ eigenfunctions. Here one has the analogue of corollary 19 by the same proof.

Theorem 22. In the topology of $\overline{\mathscr{S}}_{0}$ one has

$$
\lim _{y \rightarrow D} \frac{V_{r_{0}}(x, y)}{V_{r_{0}}(e, y)}=h^{D}(x)
$$

uniformly on compacts sets.

The Martin compactification at $r_{0}$ is the $G$-space $\bar{X} S F$.

The following appears as a purely geometric corollary of the methods developped above.

Corollary 23. Suppose $Q$ is a parabolic subgroup of $G$. Then $Q \supset N(I)$ if and only if $Q$ contains a minimal parabolic contained in $P(I)$. If $D^{I} \subset Q$, then $P(I) \subset Q$.

Proof. For the sufficiency condition, let $g B g^{-1}$ be a minimal parabolic such that $g B g^{-1} \subset P(I), g B g^{-1} \subset Q$. One has $N(I) \subset B \subset g^{-1} P(I) g$, hence $g^{-1} P(I) g=P(I), g \in$ $P(I), Q \supset g B g^{-1} \supset g N(I) g^{-1}=N(I)$.

For the necessity, one consider the twisted action $S_{g}^{0}$ on $\mathscr{F}$. If $N(I) \subset Q$, then $N(I)$ leaves invariant the closed subset $\hat{Q} \subset \mathscr{F}$ of minimal parabolic subgroups contained in $Q$. The fixed-point property of Schauder-Tychonoff for $N(I)$ [Fu2] implies the existence of a positive measure $v$ on $\hat{Q}$, such that, for some exponential $c(u)$ on $N(I)$ one has

$$
S_{u}^{0} v=c(u) v[u \in N(I)]
$$

The formula $h(x)=\int P^{1 / 2}(x, b) d v(b)$ gives now an eigenfunction $h$ such that $h\left(u^{-1} x\right)=c(u) h(x)[u \in N(I), x \in X]$.

If one can show that $c(u)=1$, then the new eigenfunction

$$
h^{\prime}(x)=\int h(k \cdot x) d m_{I}(k)
$$


will be $K^{I}$-invariant and will remain $N(I)$-invariant because $\left(k u k^{-1} \in N(I)\right.$ if $k \in K^{I}, u \in N(I)$. Hence $h^{\prime}$ will be $D^{I}$-invariant. Because of the uniqueness of such a function, as stated in the theorem, one will have $h^{\prime}=h_{I}$. But the representing measure of $h^{\prime}$ on $\mathscr{F}$ is $\int \delta_{k} * v d m_{I}(k)=v$. Hence $m_{I}=\int \delta_{k} * v d m_{I}(k)$ which implies that the support of $v$ is contained in $P(I) / B$. On the other hand this support is contained in $\hat{Q}$; hence $\hat{Q} \cap P\left(I_{/ B} \neq \varnothing\right.$. It follows that $Q \supset g B g^{-1}$ for some $g \in P(I)$. If $D^{I} \subset Q$, the set $\hat{Q}$ is also $K^{I}$-invariant, hence $\hat{Q} \supset P(I) / B$ which implies $Q \supset P(I)$.

In order to show $c(u)=1$, one consider the cone $\mathscr{C}$ of positive $\lambda_{0}$-eigenfunctions and the set $\mathscr{E}$ of exponentials $c^{\prime}$ on $N(I)$ such that there exist an eigenfunction $h^{\prime}$ with $h^{\prime}\left(u^{-1} x\right)=c^{\prime}(u) h^{\prime}(x)[u \in N(I), x \in X]$.

The group $P(I)$ acts by conjugacy on the normal subgroup $N(I)$ and also on the set $\mathscr{C}$ because $P(I)$ acts on $\mathscr{C}$ by left-translations. The compactness of the base of the cone $\mathscr{C}$ implies the compactness of $\mathscr{E}$ and the relative-compactness of the $P(I)$-orbits of elements of $\mathscr{E}$. There is a unique relatively compact $P(I)$-orbit on the vector space of exponentials on $N(I)$, that is to say the orbit of 1 . This shows $\mathscr{E}=\{1\}$ and in particular $c(u)=1$.

\section{III - THE CASE OF LOCAL FIELDS.}

In the results described above the field $\mathbb{R}$ can be replaced by a local field $F$ of any characteristic, that is to say a commutative locally compact and non discrete field. One describe very briefly here the corresponding situation.

Denote by $G_{a}$ a semi-simple algebraic group which is simply connected and defined over the field $F$ by $G$ the group of its $F$-rationnal points. It is supposed that $G_{a}$ has no $F$-anisotropic part, hence in particular $G$ is locally compact, unimodular, non amenable, compactly generated and Zariski-dense in $G_{a}$ [see Mar]. In order to simplify the notations, one will refer to $G$ instead of $G_{a}$ as a semi-simple group.

In general an algebraic subgroup of $G_{a}$ will be denoted $H_{a}$, and the set of its $F$. rational points by $H_{F}$. Denote by $B_{a}$ some minimal $F$-parabolic subgroup and $B=B_{a} \cap G$. Then $G_{a} /_{a}$ is a projective variety on which $G_{a}$ acts by projective transformations [Bor] ; furthermore, the set of its $F$-rational points can be identified with the homogeneous space $G / B=\mathscr{F}$, that is to say the set of minimal $F$-parabolic subgroups. This compact homogenous $\mathscr{F}$ space will be 
called the Furstenberg boundary of $G$. It follows from [Mar ; lemma II, 3.1] that $\mathscr{F}$ is a boundary in the sense of [Fu1]. Furthermore it plays the same role as plays the usual Furstenberg boundary (real field case) in the context of bounded $p$-harmonic functions. More precisely, one considers a maximal compact subgroup $K$ of $G$ which is transitive on $\mathrm{G}_{/ B}$ and the unique $K$ invariant probability measure $m$ on $\mathrm{G}_{/ B}$. If $G_{a}$ is simply connected such a $K$ exists [Br-Ti]. It follows from [Br-Ti] that the convolution algebra of $K$-biinvariant functions is commutative. If $p$ is a given $K$-bi-invariant probability measure on $G$, then it follows from the proofs given in [Gu2] that every bounded solution $f$ of the equation $f * p=f$ can be uniquely written in the Poisson form

$$
f(g)=g \cdot m(f)=\int f(b) P(g, b) d m(b)
$$

with $f \in \mathbb{L}^{\infty}(G / B)$ and

$$
P(g, b)=\frac{d g \cdot m}{d m}(b)
$$

Denote by $\mathscr{S}$ the space of closed subgroups of $G$ with the usual topology [Bou], by $\mathscr{S}_{0}$ the G-orbit of $K$ in $\mathscr{S}$. This gives an embedding $i$ of $X=G / K$ in $\mathscr{S}$ :

$$
i(g .0)=g K^{-1}
$$

hence a compactification $\overline{\mathscr{S}}_{0}$ of $X$.

The map $i$ is well defined because $K$ is equal to its normaliser. On the other hand the map $g \rightarrow g$. $m$ gives also an embedding $i$ of $X$ into the space $\mathscr{P}(G / B)$ of probability measures on $G_{/ B}$ because the stabiliser of $m$ is compact and equal to $K$ by maximality of $K$. One denotes by $\bar{X} S F$ the closure of $i(X)$ in $\mathscr{P}\left(G_{/ B}\right)$ with respect to the weak topology. The first problem is to compare the two compactifications of $X$ obtained in this way and to describe their elements.

Denote, as in part II

$$
\Phi_{0}(g)=\int\left(\frac{d g m}{d m}\right)^{1 / 2}(b) d m(b) .
$$

If $p$ is a $K$-bi-invariant and well behaved probability measure on $G$ one has again

$$
r_{0}=\lim _{n} p^{n}(e)^{1 / n}, \Phi_{0} * p=r_{0} \Phi_{0} .
$$

It follows from [Gu2, Mac] that the minimal solutions of the equation $f * p=r_{0}$ $f$ are uniquely given by the formula 


$$
f(g)=\int P^{1 / 2}(g, b) d v(b)
$$

where $v$ is a positive measure on $G / B$. The second problem is the following : given $D \in \overline{\mathscr{S}}_{0}$ does there exist a unique positive ro-eigenfunction $h^{D}$ such that

$$
\begin{aligned}
& h^{D} * p=r_{0} h^{D} \\
& \forall d \in D: \delta_{d} * h^{D}=h^{D} .
\end{aligned}
$$

Finally, from [Gu1] one considers as in the end of part II, the potential kernel of $p$ :

$$
V_{r}=\sum_{0}^{\infty} r^{n} p^{n} \quad\left(r \geq r_{0}\right)
$$

The third question is the identification of the cluster values of the Martin kernel $\frac{V_{r}(, y)}{V_{r}(e, y)}$ when $y$ escapes to infinity and the description of the corresponding compactification of $X$ when $r=r_{0}$ or $r>r_{0}$. This is the problem of Martin compactification of $X$. The answers to the three questions (the third when $r=r_{0}$ ) are parallel to the real field case at least if $G_{a}$ is simply connected and split over $F$. Up to natural modifications, the answers are contained in the statements of theorems 13,14 of part I, theorems 5, 5' and 19, 20 of part II. The definitions and proofs are close to the real field case but, due to rationality questions, the extension is non trivial.

The situation for $r>r_{0}$ is also considered at the end of part IV and it is conjectured that the descriptions of the Martin compactifications in the real and in the ultrametric situation should be similar.

If $G_{a}$ is split over $F$, explicit calculations are possible whereas in the general case on has to use the informations of $[\mathrm{Br}-\mathrm{Ti}]$. The key fact is the commutativity of the algebra of $K$-bi-invariant functions. Finally the Martin compactification of $X$ at $r_{0}$ is the space $\bar{X} S F$ which can be called the Satake-Furstenberg compactification of $X$.

In the case $G_{a}$ simply connected and $F$-split the notions introduced in parts I, II have a simple corresponding meaning and analogous properties are valid. For example the subgroup $A$ is the set of $F$-rational points of a maximal $F$-split torus $A_{a}$ of $G_{a}, B$ is the set of $F$-rational points of a Borel subgroup $B_{a} \supset A_{a}, N$ is the set of $F$-rational points of the unipotent radical of $B_{a}$ and $B=A N$. Denote by $\Sigma$ the set of roots of $A_{a}$ in $\mathscr{F}_{a}, \Sigma^{+}$the set of positive roots, $\Delta \subset \Sigma^{+}$the set of simple roots, $X^{+}$the set of characters of $A_{a}$ positive with respect to $\Sigma^{+}$, by $W$ the Weyl group. If $\pi$ denotes a fixed uniformizer of the local field $F$, one can define the subgroups $A^{0}$ and $\hat{A}^{0}$ of $A$ by the formulae 


$$
\begin{aligned}
& A^{0}=\left\{a \in A ; \chi(a) \in \pi^{Z} ; \forall \chi \in X\right\} \\
& \hat{A}=\left\{a \in A ; \chi(a) \in \pi^{N}, \forall \chi \in X^{+}\right\} .
\end{aligned}
$$

Given a Chevalley basis of the Lie algebra of $G$, the valuation of $F$ allows to define a compact subgroups $U$ of $N A$ such that $K=U W U$ is a maximal compact subgroup transitive on $\mathscr{F}=G / N A$ [Mac]. Then one has the Iwasawa and Cartan decompositions $G=K A^{0} N, G=K A K$. If $I \subset \Delta$ one defines $A(I)$ to be the set of $F$-rational points of the subtorus of $A_{a}$ defined by the equations $\alpha_{i}(a)=1$ ( $\left.\alpha_{i} \in I\right)$. One defines $Z(I)$ to be the centraliser of $A(I)$ in $G, G^{I}$ to be the commutator subgroup of $Z(I), P(I)=Z(I) N$. Then the Zariski closure $G_{a}^{I}$ of $G^{I}$ is semi-simple and simply connected ; furthermore $Z(I)=G^{I} A$ and $P(I)$ is the semi-direct product of $Z(I)$ and its unipotent radical $N(I)$. The orbit of $B$ in $\mathscr{F}$ can be identified with the Furstenberg boundary $\mathscr{F} I$ of $G^{I}$. Furthermore, the explicit form of $K$ shows that $K^{I}=G^{I} \cap K$ is a maximal compact subgroup of $G^{I}$ which is transitive on $\mathscr{F} I$. Hence the $K^{I}$-invariant measure $m_{I}$ on $\mathscr{F} I$ is well defined and $D^{I}=K^{I} N(I)$ is a maximal distal subgroup of $G$. In these notations the theorems 13, 14 of part I, theorems 5, 5' and 19, 20 of part II are valid. In general one can expect the following results to be valid.

Theorem 1. For every $D \in \overline{\mathscr{S}}_{0}$, there exist a unique measure $m^{D}$ on $\mathscr{F}=G / B$ which is $D$-invariant. The subgroup $D$ is distal maximal and its normalizer $R^{D}$ is a maximal amenable subgroup. The map $D \rightarrow m^{D}$ is a G-equivariant isomorphism between $\bar{Y}_{0}$ and $\bar{X} S F$.

Theorem 2. For every $D \in \overline{\mathscr{S}}_{0}$ there exist a unique normalised ro-eigen function $h^{D}$ of $p$ which is D-left-invariant. Furthermore, in the topology of $\mathscr{S}_{0}$ :

$$
\lim _{y \rightarrow D} \frac{G_{r_{0}}(x, y)}{G_{r_{0}}(0, y)}=h^{D}(x) .
$$

Hence the Martin compactification at $r_{0}$, relative to $p$ is $G$-isomorphic to $\bar{X} S F$.

\section{IV - THE MARTIN COMPACTIFICATION OF $X\left(\lambda<\lambda_{0}\right)$.}

The situation of the Laplacian will be only considered at the end.

\section{1) The compactification $\bar{X} C S F$.}

Consider the set $A^{*}$ of exponential functions on $A$. If $\alpha$ is the Lie algebra of $A$, the Killing form <., $>$ on $\alpha$ identifies the exponential $\ell \in A^{*}$ with a vector $v(\ell) \in a$. The corresponding norm on $a$ or $A^{*}$ will be denoted by the same notation : 
$|v(\ell)|=|\ell|$. One denotes by $A_{+}^{*}$ the subset of $A^{*}$ obtained in this way from the closed Weyl chamber $\bar{\alpha}^{+}$by this identification. In the same way, if $I \subset \Delta, A_{+}^{*}(I)$ will denote the subset of $A_{+}^{*}$ corresponding to the closed facet $\bar{a}+(I) \subset \bar{a}^{-}$. Consider $v \in \overline{\mathbf{a}}^{+}(\|v\|=1)$ and $v_{\infty}=\lim _{t \rightarrow+\infty} e^{t v} .0 \in \partial \bar{X} C$; the subset of $\partial \bar{X}^{C}$ corrresponding to directions in $\bar{a}^{+}\left(\right.$resp. $\left.a^{+}\right)$will be denoted $\bar{a}_{\infty}^{+}$(resp. $a_{\infty}^{+}$). More generally one consider the facet at infinity $a_{\infty}^{+}$associated to $\alpha^{+}(I)$ and the closed facet at infinity $\bar{a}_{\infty}^{+}$which corresponds to the directions of $\bar{a}+(I)$ [see BalGr-Sc].

If $a(g)$ denotes the $A$-component of $g$ in the Iwasawa decomposition $G=K A N$, the (normalised) Busemann function $A\left(x, v_{\infty}\right)\left(x \in X, v_{\infty} \in \overline{\mathrm{a}}_{\infty}^{+}\right)$can be expressed as

$$
A\left(g .0, v_{\infty}\right)=-<v, \log a\left(g^{-1}\right)>.
$$

In other words, if $\ell_{\infty} \in \overline{\mathrm{a}}_{\infty}^{+}$is the point at infinity in $\partial \bar{X}^{C}$ associated with $\ell \in A_{+}^{*}$, the functions

$$
A\left(g .0, \ell_{\infty}\right) \text { and } \log \ell\left[a\left(g^{-1}\right)\right]
$$

are proportionnal. The same property remains true of the functions $A(g .0, z)$ and $\log \sigma_{\ell}\left(g^{-1}, k\right)$ if $z=k . \ell_{\infty} \in \partial \bar{X}^{C}(k \in K)$. One denotes by $\mathscr{C}$ the projective space of the functions $\log \sigma_{\ell}^{k}$, of the argument $g$, with $\sigma_{\ell}^{k}\left(g^{-1}\right)=\sigma_{\ell}\left(g^{-1}, k\right), \ell \in A_{+}^{*}$, $k \in K$. These functions are normalised by the condition $\sigma_{\ell}^{k}(e)=1$ so that $G$ acts naturally on $\mathscr{C}$ by left translation and normalisation and $\mathscr{C}$ is a $G$-space. Because $\partial \bar{X}^{C}$ is $G$-equivariantly isomorphic to the set of (normalised) Busemann functions one has the

Proposition 1. The map $(\ell, k) \rightarrow k . \ell_{\infty}\left(k \in K, \ell \in A_{+}^{*}\right.$ defines a $G$-equivariant homeomorphism between the projective space $\mathscr{C}$ and the conical boundary $\partial \overline{\mathrm{X}} \mathrm{C}$.

One denotes by $B(z)$ the stabiliser in $G$ of $z \in \partial \bar{X} C$; in particular if $z \in \mathrm{a}_{\infty}^{+}(I)$, the subgroup $B(z)$ is equal to the standard parabolic subgroup $P(I)$. 
Definition 2. The $G$-space of pairs $(D, z) \in \overline{\mathscr{T}}_{0} \times \bar{X}^{C}$ such that $D$ is contained in the stabiliser of $z$ will be denoted $\bar{X} C S F$.

Clearly the diagonal embedding $j$ of $X$ into $\overline{\mathscr{S}}_{0} \times \bar{X}^{C}$ is a $G$-equivariant homeomorphism of $X$ with the subset $j(X)$ of $\overline{\mathscr{S}}_{0} \times \bar{X}^{C}$.

Proposition 3. Consider the diagonal embedding jof $X$ into $\overline{\mathscr{Y}}_{0} \times \bar{X}^{C}$. The closure $j(\bar{X})$ is equal to $\bar{X} C S F$.

The proof is based on the

Lemma 4. Suppose $D \in \partial \overline{\mathscr{Y}}_{0}, z \in \partial \bar{X}^{C}$ and $B(z)$ is the stabiliser of $z$. Then the condition $D \subset B(z)$ is equivalent to the condition that $z$ belongs to the closed facet at infinity associated with the parabolic subgroup $P(D)$.

Proof. It follows from part II and the fact that $B(z)$ is a parabolic subgroup that the condition $D \subset B(z)$ implies $P(D) \subset B(z)$. If $D=D^{I}$, this gives $B(z)=P(J)$ for some $J \supset I$, hence $z \in \mathrm{a}_{\infty}^{+}(J) \subset \bar{\alpha}_{\infty}^{+}(I)$. The direct implication follows from $G$ equivariance. The converse is clear from $G$-equivariance.

Proof of the proposition. The stabiliser of $i^{\prime}(x) \in \overline{\mathscr{S}}_{0}$ and $x \in \bar{X}^{C}$ are equal to $g \mathrm{Kg}^{-1}$ if $x=g .0$. The definition of the convergence

$$
\lim _{n} g_{n} K g_{n}^{-1}=D \in \overline{\mathscr{S}}_{0}
$$

and the fact that $\bar{X} C$ is a $G$-space imply $D \subset B(z)$ if $z=\lim _{n} g_{n} .0 \in \bar{X}^{C}$.

Hence $j(X) \subset \bar{X} C S F$.

In order to show the converse one can suppose by $G$-equivariance and the above lemma : $D=D^{I}, B(z) \subset B(J), z \in \mathrm{a}_{\infty}^{+}(J)$ with $I \subset J$. If $v$ is a unit vector of $\bar{a}^{+}$with direction $z \in \mathrm{\alpha}_{\infty}^{+}(J) \subset \overline{\alpha_{\infty}^{+}(I)}$, one consider some $I$-canonical sequence $a_{n} \in A^{+}(I)$ such that the sequence $v_{n}$ of unit vectors in $a^{+}(I)$ given by $v_{n}=\frac{\log a_{n}}{\left\|\log a_{n}\right\|}$ converges towards $v \in \overline{\mathrm{a}}^{+}$.

Then : $\quad \lim _{n} a_{n} K a_{n}^{-1}=D^{I}$

$$
\lim _{n} a_{n} \cdot 0=\lim _{t \rightarrow+\infty} e^{t v} \cdot 0=z
$$

hence

$$
(D, z) \in j(\bar{X}) \text {. }
$$


Corollary 5. Considerthe point $\left(D^{I}, z\right) \in \partial \bar{\varphi}_{0} \times \partial \bar{X}^{C}$ with $z$ in the facet $a_{\infty}^{+}(J)(I \subset J)$. Then the fiber of $\bar{X} C S F$ over $z$ is naturally isomorphic to the Satake-Furstenberg compactification of $X(J)$. The fiber of $\bar{X} C S F$ over $D^{I}$ is the closed facet $\bar{a}_{\infty}^{+}(I)$.

Proof. The second assertion is contained in the above lemma. In order to obtain the first assertion, one notice that the condition $z \in \mathrm{a}_{\infty}^{+}(J)$ implies $B(z)=P(J)$, hence the Langlands decomposition $P(J)=M(J) A(J) N(J)$ implies $D=[D \cap$ $M(J)] N(J)$. It follows from the end of part I that the map $D \rightarrow D \cap M(J)$ is a $G^{J}$ equivariant homeomorphism of the fiber over $z$ onto the Satake-Furstenberg compactification of $X(J)=M(J) / K^{J}$ [see also Bor-Ti1].

Corollary 6. Consider $\left(D^{I}, z\right) \in \bar{X}^{C S F} \quad\left(z \in \mathrm{a}_{\infty}^{+}(I)\right.$. The stabiliser of $\left(D^{I}, z\right)$ is $R^{I}$ and $\bar{X} C S F$ is the disjoint union of the orbits of points of the form $\left(D^{I}, z\right)\left(z \in \mathrm{a}_{\infty}^{+}(I)\right.$, $I \subset \Delta)$. The closure of the orbit of $\left(D^{I}, z\right)$ in $\bar{X} C S F$ is the union of the orbits of the points of the form $\left(D^{J}, z\right)$ with $J \subset I$.

Proof. If $g$. $\left(D^{I}, z\right)=\left(D^{I}, z\right)$, then $g D^{I} g^{-1}=D^{I} g \cdot z=z$. The first relation gives $g \in R^{I}$. But $R^{I} \subset P(I) \subset B(z)$, hence $R^{I}$ stabilises $z$, proving the first claim.

Observe that the closure of the orbit of $D^{I}$ in $\overline{\mathscr{S}}_{0}$ is equal to the union of the orbits of the points $D^{J} \in \overline{\mathscr{S}}_{0}$ with $J \subset I$. This is a compact $G$-invariant subset $\overline{\mathscr{Y}}_{0}(I)$ of $\overline{\mathscr{Y}}_{0}$. For $z \in \overline{\mathbf{a}}_{\infty}^{+}(I)$ fixed one, construct a $G$-equivariant map $\varphi_{z}$ from $\overline{\mathscr{S}}_{0}(I)$ to $\bar{X}^{C S F}$ as follows : if $D \in \overline{\mathscr{Y}}_{0}(I), D=g D^{J} g^{-1}, J \subset I$ one set $\varphi_{z}(D)=(D, g . z)$ $=g .\left(D^{J}, z\right)$. Because $z \in \overline{\mathrm{a}}_{\infty}^{+}(I) \subset \overline{\mathrm{a}}_{\infty}^{+}(J)$, one has $\left(D^{J}, z\right) \in \bar{X}^{C S F}, \varphi_{z}(D) \in \bar{X} C S F$. The map $\varphi_{z}$ is well defined because the stabiliser of $\left(D^{J}, z\right)$ is equal to the stabiliser of $D^{J}$. In order to check the continuity of $\varphi_{z}$, consider a sequence $\left(D_{n}, g_{n}\right)$ such that $\lim _{n} D_{n}=D, D_{n}=g_{n} D^{J} g_{n}^{-1}, D=h D h^{-1}$; one has to show $\lim _{n} g_{n} . z=h . z$. One can suppose $h=e$ and, from the compactness of the orbit of $z$ :

$\lim _{n} g_{n} . z=z^{\prime}$. Because $D_{n}$ stabilises $g_{n} \cdot z, D$ stabilises $\lim _{n} g_{n} \cdot z=z^{\prime}$, hence $D \subset B\left(z^{\prime}\right)$.

From the end of part II one has $P(D)=P(I) \subset B\left(z^{\prime}\right)$, hence $z^{\prime} \in \bar{\alpha}_{\infty}^{+}(I) \subset \bar{\alpha}_{\infty}^{+}$. But the orbit of $z \in \overline{\mathrm{a}}_{\infty}^{+}$is compact and intersect $\overline{\mathrm{a}}_{\infty}^{+}$in only one point, hence $z^{\prime}=z$. Notice that $\varphi_{z}$ is injective because the stabiliser of $\varphi_{z}(D)$ is $R(D)$ and this subgroup determines $D$. It follows that $\varphi_{z}$ is a $G$-equivariant homeomorphism of $\overline{\mathscr{S}}_{0}(I)$ onto its range which is equal to the orbit-closure of $\left(D^{I}, z\right)$. The structure of this orbit closure follows. 
2) A class of functions on $X$ and its relation to $\bar{X} C S F$.

Consider for $D \in \partial \overline{\mathscr{I}}_{0}, \ell \in A^{*} b \in \mathscr{F}, k \in K$ the following measures and functions (see II) :

$$
\begin{aligned}
& P_{\ell}(g, b)=\sigma_{\ell}\left(g^{-1}, b\right)=\ell^{-1}\left[a\left(g^{-1} k\right)\right] \\
& m_{*}^{D}=S_{g}^{0} m_{I} \text { if } D=g D^{I} g^{-1} \\
& h_{\ell, D}(g)=\int P_{\ell}(g, b) d m_{*}^{D}(b) \\
& h_{\ell, I}(g)=\int P_{\ell}(g, b) d m_{I}(b) .
\end{aligned}
$$

The modular function of $A N$ is denoted $\delta$. In standard notations from [He], $\delta(a)=e^{2 \rho}(a)$ if $a \in A$.

Definition 7. The exponential $\ell \in A^{*}$ is said to be $I$-adapted if one has $\ell=\delta^{1 / 2} \ell^{+}$ with $\ell^{+} \in A_{+}^{*}$ and $\ell^{+}\left(A^{I}\right)=1$. One denotes by $\Gamma$ the subset of pairs $(\ell, D) \in A^{*} \times \bar{\varphi}_{0}$ such that $\ell$ is $I$-adapted if $D$ is conjugate to $D^{I}$. One denotes by the set of functions he,D with $(\ell, D) \in \Gamma$.

Proposition 8.Suppose $(\ell, D) \in \Gamma$.Then

$$
h_{\ell, D}(g)=h^{D}(g) \sigma_{\ell+}\left(g^{-1}, b\right)
$$

where $b$ is any point in $F$ corresponding to a minimal parabolic subgroup contained in $P(D)$. In particular:

$$
S_{g} h_{\ell, D}=h_{\ell, g} D g^{-1}
$$

and the stabiliser of he,Dis $R(D)$.

Proof. A calculation with the Langlands decomposition of $P(I)$ gives that $\sigma_{\ell^{+}}(g, b)$ depends only of $g$ and of the projection of $b$ on $G / P(I)$ because $\ell^{+}\left(A^{I}\right)=1$. But $\sigma_{\ell}=\sigma_{\delta 1 / 2} \sigma_{\ell^{+}}$and $m_{*}^{D}$ is concentrated on a fiber of $G_{/ B}$ above $G / P(I)$. It follows :

$$
\begin{aligned}
h_{\ell, D}(g) & =\sigma_{\ell^{+}}\left(g^{-1}, b\right) \int \sigma_{\delta 1 / 2}\left(g^{-1}, b\right) d m_{*}^{D}(b) \\
& =\sigma_{\ell^{+}}\left(g^{-1}, b\right) h_{D}(g)
\end{aligned}
$$

with $b$ in the support of $m_{*}^{D}$; this support corresponds to the set of minimal parabolics contained in $P(D)$. 
The above formula shows that

$$
S_{g} h_{\ell, D}=h_{\ell g D g^{-1}}
$$

because $S_{g} h_{D}=h_{g D^{-1}}$ and the cocycle property of $\sigma_{\ell^{+}}(g, b)$.

In order to prove that the stabiliser of $h_{\ell, D}$ is $R(D)$ one can restrict to $D=D^{I}$.

The support of the measure $g . m_{I}$ is the support of the representing measure of $S_{g} h_{\ell, I}$. If $S_{g} h_{\ell, I}=h_{\ell, I}$ the fact that $P_{\ell}(g, b)$ are minimal eigenfunctions of the Laplacian when $\ell^{+} \in A_{+}^{*}$, [Ka, Gu2] gives that the supports of $g . m_{I}$ and $m_{I}$ an equal : $g \in P(I)$. From the formula

$$
h_{\ell, I}(g)=h_{I}(g) \sigma_{\ell^{+}}\left(g^{-1}, b\right)
$$

and the cocycle property of $\sigma_{\ell^{+}}$one gets that $S_{g} h_{I}=h_{I}$ and from part II, $g \in R^{I}$.

Theorem 9. $\Gamma$ is a closed subset of $A_{+}^{*} \times \partial \overline{\mathscr{Y}}_{0}$ and the map $(\ell, D) \rightarrow h_{\ell, D}$ is $a$ homeomorphism between $\Gamma$ and $\nVdash$.

Proof. Suppose $\lim \ell_{n}=\ell \in A^{*} \lim D_{n}=D \in \overline{\mathscr{S}}_{0}$ with every $D_{n}$ conjugate to $D^{I}$. Then from part $I D$ is a conjugate of $D^{J}$ with $J \subset I, A^{J} \subset A^{I}$. The condition $\ell_{n}^{+}\left(A^{I}\right)=1$ gives $\ell_{n}^{+}\left(A^{I}\right)=1=\ell^{+}\left(A^{J}\right)$. Hence $\Gamma$ is closed in $A^{*} \times \overline{\mathscr{S}}_{0}$. The continuity of the map $(\ell, D) \rightarrow h(\ell, D)$ is clear from the formula of the proposition above and the continuity of the map $D \rightarrow h_{D}$. In order to show the injectivity of the map $(\ell, D) \rightarrow h_{\ell, D}$, one observe that if $h_{\ell, D}$ is given, then its stabiliser $R(D)$ is known ; $D$ is also known because it is the maximum normal subgroup of $R(D)$ where the exponentials of $R(D)$ are trivial. Then the formula $h_{\ell, D}(g)=h_{D}(g) \sigma_{\ell^{+}}\left(g^{-1}, b\right)$ implies that $\sigma_{\ell^{+}}\left(g^{-1}, b\right)$ is known for $b$ corresponding to a parabolic subgroup contained in $P(D)$.

Hence the map $(\ell, D) \rightarrow h_{\ell, D}$ is bijective and continuous. Because $\Gamma$ is the union of the interiors of compact subsets, this map is bi-continuous.

Definition 10. For $(\ell, D) \in \Gamma, D=g D^{I} g^{-1}$, one defines $z(\ell, D) \in \partial \bar{X} C$ and $\Lambda(\ell, D) \in$ $\bar{X} C S F$ by $z(\ell, D)=g \cdot \ell_{\infty}^{+}, \Lambda(\ell, D)=g .\left(D, \ell_{\infty}^{+}\right)=[D, z(\ell, D)]$.

Remark. The element $g$ is defined modulo $R^{I}$ but $z(\ell, D)$ is well defined because one has $P\left(\ell_{\infty}\right) \supset P(I) \supset R^{I}$ if $\ell^{+}\left(A^{I}\right)=1$ hence $v\left(\ell^{+}\right) \in \overline{\mathrm{\alpha}}^{+}(I), \ell_{\infty}^{+} \in \overline{\mathrm{a}}_{\infty}^{+}(E)$. 
Corollary 11. For any $c>0$, denote

$$
\begin{aligned}
& \Gamma_{c}=\left\{(\ell, D) \in \Gamma ;\left\|\ell_{\infty}^{+}\right\|=c\right\} \\
& \mathscr{X}_{c}=\left\{h \ell, D ;\|\ell, D\| \in \Gamma, \| \ell_{\infty}^{+} \mid=c\right\} .
\end{aligned}
$$

The restriction of the map $\Lambda$ to $\Gamma_{c}$ is a homeomorphism between $\Gamma_{c}$ and $\partial \bar{X} C S F$. If $\Gamma_{c}$ is identified with $\varkappa_{c} \subset \mathscr{X}, \Lambda$ is a G-equivariant homeomorphism between $\mathscr{x}_{c}$ and $\bar{X}$ CSF.

Remark. Denote by $A_{+}^{*}(I)$ the set of exponentials in $A_{+}^{*}$ which are identified with elements of $\bar{a}^{+}(I)$ in the duality with respect to the Killing form. In the above statements the property of the map $\ell \rightarrow v(\ell)$ which is essential is the fact that its restrictions to any $A_{+}^{*}(I)$ is a homeomorphism onto $\bar{a}+(I)$. In particular if $p$ is a $K$-bi-invariant and well behaved probability measure on $G$, its Laplace transform $\hat{p}(\ell)\left[\ell \in A^{*}\right]$ has the following properties :

$$
\begin{aligned}
& \log \hat{p} \text { is } C^{1} \\
& \log \hat{p} \text { is strictly convex. } \\
& \hat{p}\left(\delta^{1 / 2} \ell \circ w\right)=\hat{p}\left(\delta^{1 / 2} \ell\right)
\end{aligned}
$$

for any $\ell \in A^{*}, w \in W$ [see Gu1].

Furthermore the minimal eigenfunctions are parametrised by $A_{+}^{*} \times \mathscr{F}$ as in the case of Laplacian [Fu2, Gu1, Gu2].

It follows that the Legendre duality with respect to the function of the argument $\ell$ given by $\log \hat{p}^{\gamma}\left(\delta^{1 / 2} \ell\right)\left(\ell \in A_{+}^{*}\right)$ can play the role of the map $\ell \rightarrow v(\ell)$ from $A_{+}^{*}$ to $\bar{a}+$. It is easily be shown that the above results extend to this situation. In order to stress the main facts and to prepare the way for a general approach one consider below this more general situation and one recall the definition $V_{r}=\sum_{0}^{\infty} r^{n} p^{n}\left(r \geq r_{0}\right)$.

\section{3) The Martin boundary of $X$.}

The condition $\ell$ adapted $\left.\left[\ell=\delta^{1 / 2} \ell^{+}, \ell^{+}\left(A^{I}\right)\right]=1, \ell^{+} \in A_{+}^{*}\right]$ plays a role throught the following minimum property with constraints. 
Proposition 12. Suppose $p$ is a $K$-bi-invariant and well behaved probability measure and $\ell=\delta^{1 / 2} \ell^{+}$is $I$-adapted. Then, if $f \in A^{*}$ is such that the restrictions of $f$ and $\ell$ to $A(I)$ are equal, one has $\hat{p}(f) \geq \hat{p}(\ell)$ with equality if and only if $f=\ell$.

Proof. Write $\bar{f}, \bar{f}$, for the restrictions of $f$ to $A^{I}, A(I)$ and by abuse of notations : $f=\bar{f} \bar{f}$.

One has, if $g \in P(I), b \in P(I) / B$

$$
\left.P_{f}(g, b)=P_{\tilde{f}} \widetilde{g}, b\right) \bar{f}(a)
$$

where $g \in P(I)$ is written in Langlands's form $\mathscr{G}=\tilde{g} a \eta \quad[\tilde{g} \in M(I), a \in A(I)$, $\eta \in N(I)]$.

The function $h_{f}^{b}=P_{f}(g, b)$ satisfies

$$
\begin{aligned}
& h_{f}^{b} * p=\tilde{p}(f) h_{f}^{b} \\
& h_{f}^{b} * q=\tilde{p}(f) h_{f}^{b}
\end{aligned}
$$

where in the second equation, which is valid on $P(I)$, one has $q=\pi(p)$ [see II]. If $\Phi_{\tilde{f}^{-}(\tilde{g})}=\int P_{\widetilde{f}^{-}}(\tilde{g}, b) d m_{I}(b)$

it follows

$$
\tilde{p}(f)=\int \Phi_{\tilde{f}}\left(\tilde{g}^{-1}\right) \bar{f}(a) d q(\widetilde{g}, a) .
$$

Denote by $\Phi_{I}$ the function $\Phi_{\tilde{f}}$ in the case $f=\delta_{I}^{1 / 2}$ where $\delta_{I}$ is the modular function of $A^{I} N^{I}$.

Because $\Phi_{\tilde{f}} \geq \Phi_{I}=\Phi_{\delta_{I}^{1 / 2}}$ the formula for $\hat{p}(f)$ gives

$$
\hat{p}(f) \geq \hat{p}\left(\delta_{I}^{1 / 2} \bar{f}\right) .
$$

If the restrictions of $f$ and $\ell$ to $A(I)$ are equal one has $\bar{\ell}=\bar{f}$; furthermore $\ell=\delta_{I}^{1 / 2} \bar{\ell}$ because $\ell$ is $I$-adapted, hence

$$
\hat{p}(f) \geq \hat{p}\left(\delta_{I}^{1 / 2} \bar{f}\right)=\hat{p}\left(\delta_{I}^{1 / 2} \bar{\ell}\right)=\hat{p}(\ell) .
$$

The minimum of the function $\hat{p}(f)$ under the constraint $\bar{f}=\bar{\ell}$ is attained at a unique point because of the strict convexity of $\hat{p}$. Hence the equality $\hat{p}(f)=\hat{p}\left(\delta_{I}^{1 / 2} \bar{\ell}\right)$ implies $f=\delta_{I}^{1 / 2} \bar{\ell}$. 
Theorem 13. Suppose $p$ is well behaved and $K$-bi-invariant and $\ell \in A^{*}$ is $I-$ adapted.Suppose that the positive eigen function $h$ has the following properties :

a) $h * p=\hat{p}(\ell) h$

b) $h$ is $D^{I}$-invariant

c) the ratio $\frac{h(a g)}{h(g) \ell(a)}$ is bounded for $g \in G, a \in A(I)$.

\section{Then $h$ is proportionnal to $h, I$.}

Proof. As in the proof of the proposition one restricts the equation

to the subgroup $P(I)$ :

$$
h * p=\hat{p}(\ell) h
$$

$$
h * q=\hat{p}(\ell) h \text {. }
$$

If $\bar{p}$ denotes the projection of $q$ on $M(I) A(I)$ one has

$$
h * \bar{p}=\hat{p}(\ell) h .
$$

Hence, on $M(I) A(I), h$ is a barycenter of minimals of the form $h_{f}^{b}(g)=P_{\widetilde{f}}(\widetilde{g}, b) \bar{f}(a)=P_{f}(g, b)$ with $b \in P(I) / B$ and $f \in A^{*}$ satisfies $p^{\sim}(f)=\hat{p}(\ell)$ [see II].

If one takes $\boldsymbol{g}=\tilde{\boldsymbol{g}}$ in the boundedness hypothesis of the theorem, one gets that a certain barycenter of exponentials on $A(I)$ of the form $\bar{f}$ is bounded by $C \bar{\ell}(C>0)$; This implies that this barycenter is proportionnal to $\bar{\ell}$ and every term $\bar{f}$ in it is equal to $\bar{\ell}$. The conditon that $\ell$ is $I$-adapted and $\hat{p}(f)=\hat{p}(\ell)$ gives now $f=\ell$, $P_{\ell}=P_{f}$

$$
h(g)=\int P_{\ell}(g, b) d v(b)
$$

with a certain positive measure $v$ on $P(I) / B$. Finally the $D^{I}$-invariance of $h$ gives the invariance of $v$ under the action of $K^{I}$ :

$$
v=m_{I} \quad, \quad h=h_{\ell, I} .
$$

Corollary 14. Suppose $\ell \in A^{*}$ is I-adapted and $\hat{p}(\ell)=r$. Suppose that $y_{n} \in X$ is a sequence which converges towards $D^{I} \in \overline{\mathscr{S}}_{0}$ and such that $\varlimsup_{n} \frac{V_{r}\left(a \cdot x, y_{n}\right)}{V_{r}\left(x, y_{n}\right) \ell(a)}$ is bounded when $a \in A(I), x \in X$.

Then the sequence $\frac{V_{r}\left(x, y_{n}\right)}{V_{r}\left(0, y_{n}\right)}$ converges towards $h_{\ell, I}$. 
Proof. Consider a subsequence $y_{n_{k}}$ such that $h(x)=\lim _{k} \frac{V_{r}\left(x, y_{n_{k}}\right)}{V_{r}\left(0, y_{n_{k}}\right)}$ exists. One has

$$
\begin{aligned}
& h(0)=1 \\
& h * p=r h=\hat{p}(\ell) h .
\end{aligned}
$$

Moreover $\frac{h(a x)}{h(x) \ell(a)}$ is bounded in $a \in A(I), x \in X$ and $h$ is $D^{I}$-invariant because $\lim _{n} y_{n}=D^{I}$ in $\overline{\mathscr{Y}}_{0}$. The theorem gives $h=h_{\ell, I}$. Hence the limit of $\frac{V_{r}\left(x, y_{n}\right)}{V_{r}\left(0, y_{n}\right)}$ exists and is equal to $h_{\ell, I}$.

In order to express the functions above in geometrical terms recall that the Busemann function $A(x, z)\left(x \in X, z \in \partial \bar{X}^{C}\right)$ is defined as

$$
A(x, z)=\lim _{y \rightarrow z} d(0, y)-d(x, y)
$$

where $d$ is the Riemann distance. The Busemann cocycle $\beta(g, z)$ on $G \times \partial \bar{X}^{C}$ is defined has

$$
\beta(g, z)=e^{A\left(g^{-1}, 0, z\right)} .
$$

If $v \in \overline{\mathrm{a}}^{+},|v|=1$ and $z=v_{\infty} \in \overline{\mathrm{a}}_{\infty}^{+} a \in A$, one has $A\left(a, v_{\infty}\right)=\left\langle v, \log a>\beta\left(a, v_{\infty}\right)\right.$ $=-\langle v, \log a\rangle$.

Consider an exponential $\ell$ on $A$ which is $I$-adapted $: \ell=\delta^{1 / 2} \ell^{+}$with $\ell^{+} \in A^{*}$, $\ell^{+}\left(A^{I}\right)=1$. Suppose $z \in \partial \bar{X}^{C}$ belongs to the orbit of $\ell_{\infty} \in \bar{a}_{\infty}^{+}(I)$ which is isomorphic to $G_{/ P(I)}$. If $b \in \mathscr{F}$ corresponds to a minimal parabolic subgroup of $B(z)$, then, from G-equivariance, one sees that

It follows that

$$
\sigma_{\ell^{+}}(g, b)=\beta-\|+\|(g, z) \text {. }
$$

$$
\sigma \ell(g, b)=\sigma_{\delta 1 / 2}(g, b) \beta^{-\|\ell+\|}(g, z)=P^{1 / 2}\left(g^{-1}, b\right) \beta^{-\| \ell+} \|(g, z)
$$

when $z \in \partial \bar{X} C$ is associated with $\ell^{+}$and $b$ as above.

Also $h_{\ell, D}(g)=h_{D}(g) \beta^{-\| \ell+1}\left(g^{-1}, z\right)$.

From [He] one knows that, for $\ell$ and $b$ fixed, the functions $\sigma \ell(g, b)$ are eigenfunctions of $\Delta$ with eigenvalue $\left\|\ell^{+}\right\|^{2}-\|\rho\|^{2}$.

One shall denote, for $\lambda<\lambda_{0}$ fixed, by $h_{z, D}^{\lambda}$ the $\lambda$-eigenfunction $h_{\ell, D}(g)=h_{D}(g)$ $\beta^{-}\left\|\ell^{+}\right\|\left(g^{-1}, z\right)$ with $\left\|\ell^{+}\right\|^{2}=\lambda_{0}-\lambda$.

One can summarise the above discussion in the 
Lemma 15. The minimal $\lambda$-eigenfunctions of the Laplacian are the functions of the form

$$
h_{z, b}^{\lambda}(g)=P^{1 / 2}(g, b) \beta^{-\sqrt{\lambda_{0}-\lambda}}\left(g^{-1}, z\right)
$$

where $b \in \mathscr{F}, z \in \partial \bar{X}^{C}$ are related by the condition $b \subset B(z)$. Here $P$ is the Poisson kernel and $\beta$ the Busemann cocycle of $G$.

One has the formula

$$
h_{z, D}^{\lambda}(g)=\int h_{z, b}^{\lambda}(g) d m_{*}^{D}(b)=h_{D}(g) \beta^{-\sqrt{\lambda_{0}-\lambda}}\left(g^{-1}, z\right)
$$

with $D \subset B(z)$.

One has then the statement analogous to corollary 14 for the Green kernel $G_{\lambda}$; the proof is the same.

Corollary 16. Suppose $y_{n} \in X_{0}$ is a sequence of $X$ converging to $D \in \overline{\mathscr{Y}}_{0}$ such that $\lim _{n} \frac{G_{\lambda}\left(r^{-1} x_{2} y_{n}\right)}{G_{\lambda}\left(x, y_{n}\right) h_{z, D}^{\lambda}\left(r^{-1)}\right.}$ is bounded when $r \in R(D) x \in X$. Then the sequence $\frac{G_{\lambda}\left(x, y_{n}\right)}{G_{\lambda}\left(0, y_{n}\right)}$ convergestowards $h_{z, D}^{\lambda}(x)$.

4) Calculation of the Martin compactification (case of Laplacian).

Now in order, to verify the hypothesis of the last corollary one has to estimate the Green kernel $G_{\lambda}$; this is done in the lemmas below. The basic estimate is obtained in [An-Ji].

Lemma 17. There exist two constants $A_{1}, A_{2}$ and a function $g_{\lambda}$ on $\bar{\alpha}+$ such that

$$
A_{2} g_{\lambda}(H) \leq G_{\lambda}\left(0, e^{H}\right) \leq A_{1} g_{\lambda}(H)
$$

and the ratio $\frac{g_{\lambda}\left(H_{n}-L\right)}{g_{\lambda}\left(H_{n}\right)}$ has a limit when $e^{H_{n}}$ is I-canonical, $\lim _{n} \frac{H_{n}}{\left|H_{n}\right|}=v$, $L \in \mathrm{\alpha}(I)$.

This limit is equal to $e^{\rho(L)+\sqrt{\lambda_{0}-\lambda}<\nu, L>}=h_{I}\left(e^{L}\right) \beta^{-\sqrt{\lambda_{0}-\lambda}}\left(e^{-L}, v_{\infty}\right)$.

Proof. From [An-Ji] the first result is valid with

$$
g_{\lambda}(H)=\|H\|-e \prod_{\alpha \in \Sigma_{0}^{+}}[1+\alpha(H)] e^{-\rho(H)-\sqrt{\lambda_{0}-\lambda}\|H\|}
$$

where $\Sigma_{0}^{+}$is the set of indivisible roots and 


$$
e=\frac{r+1}{2}+\left|\Sigma_{0}^{+}\right|-1 \quad\left(\lambda<\lambda_{0}\right)
$$

For $n$ large, if $L \in \mathrm{a}(I)$, one has $H_{n}-L \in \overline{\mathrm{a}}+$ because $e^{H_{n}}$ is $I$-canonical. Clearly

$$
\lim _{n} \frac{\left|H_{n}-L\right|}{\left|H_{n}\right|}=1
$$

For the ratio $\frac{1+\alpha\left(H_{n}-L\right)}{1+\alpha\left(H_{n}\right)}$ two cases occur. If $\alpha \in[E]$, then $\alpha(L)=0$ and this ratio is one ; if $\alpha \notin[E], \lim _{n} \alpha\left(H_{n}\right)=+\infty$ and $\lim _{n} \frac{1+\alpha\left(H_{n}-L\right)}{1+\alpha\left(H_{n}\right)}=1$ because $e^{H_{n}}$ is $I$ canonical.

Finally one has, from euclidean geometry

$$
\lim _{n}\left|H_{n}-L\right|-\| H_{n} \mid=-<v, L>
$$

if $\lim _{n} \frac{H_{n}}{\left|H_{n}\right|}=v$

All these remarks give :

$$
\lim _{n} \frac{g \lambda\left(H_{n}-L\right)}{g_{\lambda}\left(H_{n}\right)}=e^{\rho(L)+\sqrt{\lambda_{0}-\lambda}<v, L>} .
$$

The formulas for $h_{I}$ and $\beta\left(e^{-L}, v_{\infty}\right)$ give the final equality.

Lemma 18. Suppose $y_{n} \in X$ converges to $D^{I} \in \overline{\mathscr{S}}_{0}$. Then $y_{n}$ can be written $y_{n}=\varepsilon_{n} a_{n} .0$ where $\lim _{n} \varepsilon_{n}=e$ and $a_{n}$ is I-canonical.

Proof. One can write in the polar decomposition $y_{n}=k_{n} a_{n} .0$ with $a_{n} \in \overline{A^{+}}$. Extracting subsequences, one can suppose $a_{n}=\bar{a}_{n} \bar{a}_{n}$ with $\bar{a}_{n} J$-canonical, $\tilde{a}_{n} \in \bar{A}^{+J}, \lim _{n} \tilde{a}_{n}=\tilde{a} \in \bar{A}^{+J}, \lim _{n} k_{n}=k \in K$.

Then : $\lim _{n} \bar{a}_{n} K \bar{a}_{n}^{-1}=D^{J}$ and $k \tilde{a} D^{J} \tilde{a}^{-1} k^{-1}=D^{I}$, hence $J=I, k \tilde{a} \in R(I)$. Because $\tilde{a} \in P(J)=P(I)$ one has $k \in P(I)$. But $P(I) \cap K \supset K^{I}$ and $K^{I}$ is a maximal compact subgroup of $P(I)$; it follows $P(I) \cap K=K^{I}, k \in K^{I}$. Because $k \tilde{a} \in R(I)$ one has $\tilde{a} \in R(I) \cap A^{I}=\{e\}$. It follows that for some $\varepsilon_{n}$ with $\lim _{n} \varepsilon_{n}=e$ one has $y_{n}=\varepsilon_{n} k \bar{a}_{n} \cdot 0=\varepsilon_{n} \bar{a}_{n} k \cdot 0=\varepsilon_{n} \bar{a}_{n} \cdot 0$, hence the result. 
Lemma 19. Suppose $y_{n} \in X$ is a sequence of the form $y_{n}=\varepsilon_{n} a_{n} .0$ with $a_{n} I-$ canonical and $\lim _{n} \varepsilon_{n}=e$. Suppose $x \in X$ is written [using the Langlands decomposition of $P(I)]: x=k \eta a .0\left(k \in K^{I}, \eta \in N(I), a \in A\right)$. Then one has

$\lim _{n} \frac{G_{\lambda}\left(x, y_{n}\right)}{G_{\lambda}\left(a \cdot 0, a_{n} \cdot 0\right)}=1$

Proof. One has $G_{\lambda}\left(x, y_{n}\right)=G_{\lambda}\left(k\left(k^{-1} \varepsilon_{n}^{-1} k\right) \eta a .0, a_{n} .0\right)=G_{\lambda}\left(\varepsilon_{n}^{\prime} \eta a .0, a_{n} .0\right)$ because $k a_{n}=a_{n} k$ if $k \in K^{I}\left(\varepsilon_{n}^{\prime}=k^{-1} \varepsilon_{n}^{-1} k\right)$.

From Harnack inequality one gets

$$
\lim _{n} \frac{G_{\lambda}\left(x, y_{n}\right)}{G_{\lambda}\left(\eta a \cdot 0, a_{n} \cdot 0\right)}=1
$$

On the other hand :

$$
G_{\lambda}\left(\eta a \cdot 0, a_{n} \cdot 0\right)=G_{\lambda}\left(a \cdot 0, a_{n}\left(a_{n}^{-1} \eta a_{n}\right) .0\right)
$$

Then the relation $\lim _{n} a_{n}^{-1} \eta a_{n}=e$ is valid because $a_{n}$ is $I$-canonical and Harnack inequality imply again

$$
\lim _{n} \frac{G_{\lambda}\left(\eta a .0, a_{n} .0\right)}{G_{\lambda}\left(a .0, a_{n} .0\right)}=1
$$

hence the result.

Proposition 20. Suppose that the sequence $y_{n} \in X$ converges to $(D, z) \in \bar{X}^{C S F}$ and $r \in R(D), x \in X$. Then with $A_{1}, A_{2}$ as in lemma 17 one has

$$
\begin{aligned}
\varlimsup_{y_{n} \rightarrow\left(D_{z}\right)} \frac{G_{\lambda}\left(r^{-1} x, y_{n}\right)}{G_{\lambda}\left(x, y_{n}\right)} & \leq \frac{A_{1}}{A 2} h_{D}\left(r^{-1}\right) \beta^{\sqrt{\lambda_{0}-\lambda}}(r, z) \\
& =\frac{A_{1}}{A_{2}} h_{z, D^{\left(r^{-1}\right)}}^{\lambda}
\end{aligned}
$$

Proof. From G-equivariance one can suppose $D=D^{I}, z=v_{\infty} \in \bar{\alpha}+(I)$. From lemma 18 one can write $y_{n}$ in the form $y_{n}=\varepsilon_{n} a_{n} .0$ where $\lim _{n} \varepsilon_{n}=e$, and $a_{n}$ is $I$ canonical. If $x$ is written in the form $x=k \eta a .0\left(k \in K^{I}, \eta \in N(I), \alpha \in A\right)$ with $a=\tilde{a} \bar{a} \quad \bar{a} \in A(I), \tilde{a}^{-1} \in \bar{A}^{+I}$, one has from lemma 18

$$
\lim _{n} \frac{G_{\lambda}\left(x, y_{n}\right)}{G_{\lambda}\left(a, a_{n} .0\right)}=1
$$


Denote by $b$ the $A(I)$-part of $r \in R(I)$ in the Langlands decomposition. Then, one has also : $\lim _{n} \frac{G_{\lambda}\left(r^{-1} x, y_{n}\right)}{G_{\lambda}\left(b^{-1} a, a_{n} .0\right)}=1$.

It follows :

$$
\varlimsup_{n} \frac{G_{\lambda}\left(r^{-1} x, y_{n}\right)}{G_{\lambda}\left(x, y_{n}\right)}=\varlimsup_{n} \frac{G_{\lambda}\left(b^{-1} a, a_{n} \cdot 0\right)}{G_{\lambda}\left(a, a_{n} .0\right)} .
$$

But, if one write $a_{n}=e^{H_{n}}, a=e^{H}, b=e^{L}$ with $H_{n} \in \alpha^{+}(I), L \in \mathrm{\alpha}(I), H=\bar{H}+\widetilde{H}$ $H \in \mathrm{a}(I),-\widetilde{H} \in \overline{\mathrm{a}}^{+}(I)$ one has from lemma $17: G_{\lambda}\left(a, a_{n} .0\right) \geq A_{2} g_{\lambda}\left(H_{n}-\tilde{H}-\bar{H}\right)$, $G_{\lambda}\left(b^{-1} a, a_{n}, 0\right) \leq A_{1} g_{\lambda}\left(H_{n}-\widetilde{H}+L-\bar{H}\right)$.

Because $a_{n}$ is $I$-canonical, and $-\widetilde{H} \in \bar{\alpha}^{+I}$ one has for $n$ large

$$
H_{n}-\tilde{H}+L-\bar{H} \in \overline{\mathrm{a}}^{+} \text {. }
$$

From the lemma 17 above

$$
\lim _{n} \frac{g_{\lambda}\left(H_{n}-\tilde{H}+L-\bar{H}\right)}{g_{\lambda}\left(H_{n}-\tilde{H}-\bar{H}\right)}=e^{-\rho(L)-\sqrt{\lambda_{0}-\lambda}<v, L>}
$$

if $\lim _{n} \frac{H_{n}}{\left|H_{n}\right|}=v \in \overline{\mathrm{a}}^{+}$.

The form of $h_{D}\left(e^{L}\right)$ and of $\sigma\left(e^{L}, v_{\infty}\right)$ gives :

$$
\varlimsup_{n} \frac{G_{\lambda}\left(r^{-1} x, y_{n}\right)}{G_{\lambda}\left(x, y_{n}\right)} \leq \frac{A_{1}}{A_{2}} h_{D}\left(r^{-1}\right) \beta^{-\sqrt{\lambda_{0}-\lambda}}(r, z) .
$$

Theorem 21. For $\lambda<\lambda_{0}$, one has

$$
\lim _{n} \frac{G_{\lambda}\left(x, y_{n}\right)}{G_{\lambda}\left(0, y_{n}\right)}=h_{z, D}^{\lambda}(x)
$$

The Martin compactification of $X$ with respect to the Laplacian is equal to $\bar{X} C S F$ and the cocycle $\theta^{\lambda}[g,(D, z)]$ is equal to $h_{D}\left(g^{-1}\right) \beta^{-\sqrt{\lambda_{0}-\lambda}}(g, z)$.

The points $(D, z) \in \bar{X} C S F$ which give minimal eigen functions are the pairs such that $D$ is conjugate to a subgroup $M N$ and $D \subset B(z)$.

Proof. In order to obtain the first equality, one consider a sequence $y_{n} \in X$ such that $\lim _{n} y_{n}=(D, z) \in \bar{X}^{C S F}$.

Because of proposition 19, the hypothesis of corollary 15 are satisfied hence

$$
\lim _{n} \frac{G_{\lambda}\left(x, y_{n}\right)}{G_{\lambda}\left(0, y_{n}\right)}=h_{z, D}^{\lambda}(x)
$$


As in the corollary 11 to theorem 9 one sees that the map from $\bar{X}$ CSF into $\mathscr{H}_{c}$ given by $(D, z) \rightarrow h_{z, D}^{\lambda}\left[\sqrt{\lambda_{0}-\lambda}=c\right]$ is a homeomorphism onto its range, in particular it is injective. This gives the second assertion.

In the situation where $G$ is a semi-simple algebraic group defined a local field (see part III), the random walk framework considered above remains valid. The "conical" compactification of $\mathrm{G} / K$ is well defined [Bor-Se] as well as the Busemann function if one uses the natural metric on the Bruhat-Tits building. The validity of the content of theorem 21 in this situation depends only of the validity of an analogue of lemma 17.

In this case simple explicit formulae are available for the spherical functions and the Harish-Chandra $c$-function [Mac]. Hence the expression of the Green kernel restricted to $A . o \subset \mathrm{G} / K$ reduces to an explicit Fourier intégral on an $r$ dimensional torus. Using the methods described in [Bab2] it is easy to show that lemma 17 is valid with natural changes. Hence the same description of the Martin compactification should remain valid in the ultrametric situation.

Suppose now that $G$ is the connected component of the set of real points of a fixed $\mathbb{R}$-algebraic group, again denoted by $G$.

One can then express the minimal $\lambda$-eigenfunctions in terms of the fondamental representations of $G$ [Bor]. Consider the inverse root system of $\Sigma$ and the canonical basis defined in terms of the Killing form which allow identification of $\alpha$ and $\alpha^{*}$ :

$$
\widetilde{\alpha_{i}}=\frac{2 \alpha_{i}}{\left\|\alpha_{i}\right\|^{2}}
$$

Then if $\omega_{i}$ denotes the dual basis of $\check{\alpha_{i}}$, the Killing form has the expression :

$$
<v, a>=\sum_{i=1}^{r} \check{\alpha} \check{\alpha}_{i}(v) \omega_{i}(a)
$$

where $v, a \in a$.

The linear form $\omega_{i}$ defines a fondamental representation $\rho_{i}$ of $G$ in a finite dimensionnal real vector space $V_{i}$ [Bor]. This representation is strongly rationnal, irreducible [Bor-Ti1] and its highest weight is a rationnal multiple of $\omega_{i}$. If this weight is denoted $r_{i}^{-1} \omega_{i}$, the rationnal $r_{i}^{-1}$ is supposed to be the smallest positive rationnal having such a property. If $G$ is split over $\mathbb{R}$ and simply connected, then $r_{i}=1$. There exist a unique line in $V_{i}$ which is invariant 
under $B$ and its stabilizer is a maximal parabolic subgroup $P_{i} \supset B$. One denotes by $f_{i}$ a unit vector in this line and by $\|$. $\|$ the unique (up to a scalar) $K$-invariant scalar product in $V_{i}$ :

if $g \in P_{i}$.

$$
\rho_{i}(g) f_{i}=e^{r_{i}^{-1} \omega_{i}}(g) f_{i}
$$

In the projective space $P\left(V_{i}\right)$, the orbit of $f_{i}$ is isomorphic to $\mathrm{G} / P_{i}$ and every point in this orbit corresponds to a point in $\mathscr{F}$. Then, if $x$ is a unit vector in $V_{i}$ corresponding to $b \in \mathscr{F}$, the fondamental cocycle $\sigma_{i}(g, b)$ is defined by the formula :

$$
\sigma_{i}(g, b)=\left\|p_{i}(g) x\right\|
$$

The restriction of the Busemann function to $A$ when $z \in \overline{\mathrm{a}}_{\infty}^{+}$corresponds to a unit vector $v$ in $\overline{\mathbf{a}}^{+}$is given by

$$
A\left(a, 0, v_{\infty}\right)=\langle v, \log a>\text {. }
$$

Hence the above formula giving the Killing form leads to the following formula for the Busemann cocycle $\beta$

$$
\beta(g, z)=\prod_{i=1}^{r} \sigma_{i}^{r_{i} \check{\alpha_{i}(v)}(g, b) .}
$$

Here $|v|=1, z=k . v_{\infty}$ where $k \in K$ corresponding to $b \in \mathscr{F}$. The Poisson kernel corresponds to $v=-2 \rho$ with standard notations [He]. But from [He] : $\rho=\sum_{i=1}^{r} \omega_{i}$, hence

$$
P^{1 / 2}\left(g^{-1}, b\right)=\prod_{i=1}^{r} \sigma_{i}^{-r_{i}}\left(g^{-1}, b\right)
$$

with $r_{i}$ the rationnal defined above.

This leads to the following formula for the minimal eigenfunctions :

$$
h_{z, b}^{\lambda}(g)=\prod_{i=1}^{r} \sigma_{i}^{-r_{i}-r_{i} \sqrt{\lambda_{0}-\lambda} \ddot{\alpha_{i}}(v)}\left(g^{-1}, b\right) .
$$

where $v \in \overline{\mathbf{a}}^{+},\|v\|=1$.

One consider now the special case $G=S \ell(d, \mathbb{R})$. Then if $v=\operatorname{diag}\left(v_{1}, v_{2},, v_{d}\right) \in \alpha$ the Killing form is given by $\|v\|^{2}=2 d \sum_{i=1}^{d} v_{i}^{2}$. The fondamental roots are given by 
$\alpha_{i}(v)=v_{i}-v_{i+1}(1 \leq i \leq d-1)$, hence $\left\|\alpha_{i}\right\|^{2}=\frac{1}{d}$. The fondamental representations are the representation of $G$ in the wedge product $V_{i}=\wedge^{i} \mathbb{R}^{d}(1 \leq i \leq d)$ and $r_{i}=1$. A sequence of unit multivectors : $b_{1}=x_{1}, b_{2}=x_{1} \wedge x_{2}, \ldots b_{d-1}=x_{1} \wedge \ldots \wedge x_{d-1}$ defines a flag $b$ and the fondamental cocycles $\sigma_{i}$ are given by $\sigma_{i}(g, b)=\left|g b_{i}\right|$. From the above formulae, one gets the Poisson cocycle

$$
\sigma(g, b)=\prod_{i=1}^{d-1}\left\|g b_{i}\right\|^{-2}
$$

A vector $v \in \overline{\mathrm{a}}^{+}$defines a point $v_{\infty} \in \overline{\mathrm{a}}_{\infty}^{+} \subset \bar{X}^{C}$ and if $b \in \mathscr{F}$ corresponds to $k \in K$, one denotes by $z$ the point of $\partial \bar{X}^{C}$ defined by :

$$
z=k \cdot v_{\infty}
$$

Because $\left|\alpha_{i}\right|^{2}=\frac{1}{d}$, one has $\check{\alpha_{i}}(v)=2 d\left(v_{i}-v_{i+1}\right)$ and the Busemann cocycle $\beta(g, z)$ is now given by

$$
\beta(g, z)=\prod_{i=1}^{d-1}\left\|g b_{i}\right\|^{v_{i}-v_{i+1}}
$$

with the condition $\sum_{i=1}^{d} v_{i}^{2}=2 d$.

Hence the minimal $\lambda$-eigenfunctions are given by the formula :

$$
h_{z, b}^{\lambda}(g)=\prod_{i=1}^{d-1}\left\|g^{-1} b_{i}\right\|^{-1-\sqrt{\lambda_{0}-\lambda(}\left(v_{i}-v_{i+1}\right)}
$$

with the conditions

$$
v_{1} \geq v_{2} \geq \ldots \geq v_{d}, \sum_{i=1}^{d} v_{i}=0 \quad \sum_{i=1}^{d} v_{i}^{2}=2 d
$$

The formula $\lambda_{0}=\mid \rho \|^{2}$ leads to $\lambda_{0}=d \sum_{k=0}^{d-1}(d-2 k-1)^{2}$. 


\section{REFGRENCES}

[Ab] Abels : Distal affine transformation groups. J. Reine angew Math 299/300, 1978, p 294-300.

[An-Ji] Anker-Ji : Heat kernel and Green function estimates for semi-simple non compact Lie groups. En préparation.

[Bab1] Babillot : Potentialat in finity on symmetric spaces and Martin boundary. In "Harmonic Analysis and discrete potential theory". M. Picardello ed. Plenum press NY (1992), p 282-288.

[Bab2] Babillot : Théorie du renouvellement pour des chaines semi-markoviennes transientes. Ann. IHP 24, n 4, (1988), p 507-569.

[Bal-Gr-Sc] Ballmann-Gromov-Schroeder : Manifolds of non positivecurvature. Progress Math Birkhaüser, 1985.

[Bor] Borel : Introduction auxgroupesarithmétiques. Hermann Paris, 1965.

[Bor-Se] : Borel-Serre : Cohomologied'immeublesetdegroupesarithmétiques. Topology 15, 1976, p 211-232.

[Bor-Ti1] Borel-Tits : Groupes réductifs. Publ. Math IHES 27 (1965) p 55-150.

[Bor-Ti2] Borel-Tits : Elements unipotentsetsous-groupesparaboliquesdegroupes réductifs. I. Invent Math 12 (1971) p 95-104.

[Bou1] Bourbaki : Integration. Chap. VI-VIII. Hermann Paris 1960.

[Bou2] Bourbali : Groupes et algèbres de Lie. Chap. IV-VII.Hermann.

[Boug] Bougerol : Comportement à l'infini du noyau potentiel sur un espace symétrique. Springer Lecture Notes in Math 1096 (1984), p 90-115.

[Br-Ti] Bruhat-Tits : Groupes réductifs sur un corps local I, II. Publ. Math. IHES, $n^{\circ} 41,1972$ et $n^{\circ} 60,1984$.

[C] Cartan : Leçons sur la géométrie des espaces de Riemann. Gauthier-Villars, Paris.

[Ca] Cartier : Géométrie et analyse sur les arbres. Sém. Bourbaki 24è année 71-72, exp. 407.

[Ch] Choquet-Deny : Sur l'équation de convolution $\mu=\mu * \sigma$. CRAS Paris 250 (1960), p 779-780.

[Co-Gu] Conzo-Guivarc'h : Remarques sur la distalite dans les espaces vectoriels. CRAS 278 (1974), A p 1083-1086.

[Do] Doob : Classical potential theory and its probabilistic counter part. Grundehleren der Math, Springer 1984.

[Dy1] Dynkin : Markov processes and problems in Analysis. Proc. Int. Congr. 1962, Translations of the AMS vol. 31, ser 2, p 1-24 (1963).

[Dy2] Dynkin : Non negative eigen functions of the Laplace-Beltrami operator in certain symmetric spaces. Math USSR Isvestyia 7

[He] Helgason : Groups and geometric analysis. Vol. I, II, Academic press New York 1983.

[Fu1] Furstenberg : A Poisson formula for semi-simple groups. Annals of Math, vol. 77, 1963, p 335-386.

[Fu2] Furstenberg : Translation invariant cones of functions. Bull. AMS 71 (1965), p 272-326.

[Ge] Gérardin : On harmonic functions on symmetric spaces and buildings. Canad. Math. Soc. Conf. Proceedings 1 (1981), p 79-92.

[Gl] Glasner : Proximal flows. Springer Lectures Notes in Mathematics 517 (1976).

[Gi-Wo] Giulini-Woess : The Martin compactification of the cartesian product of two hyperbolic spaces. J. Reine ang Math 444 (1993) p 17-28. 
[Gu1] Guivarc'h : Loi des grands nombres et rayon spectral d'une marche aleatoire sur un groupe de Lie. Astérisque 74 (1980) p 47-98.

[Gu2] Guivarc'h : Sur la représentation intégrale des fonctions propres du

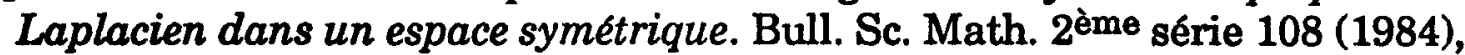
p 373-392.

[Gu-Ji-Ta] Guivarc'h-Ji-Taylor : Compactification of symmetric spaces. CRAS t. 317, ser. I, 1993, p 1103-1108.

[Gu-Ra1] Guivarc'h-Raugi : Frontière de Furstenberg, propriétés de contraction et theorèmesdeconvergence. Z. Wahr 89, 1985, p 187-242 .

[Gu-Ra2] Guivarc'h-Raugi : Propriétés de contraction d'un semi-groupe de matricesinversibles. Israël J. of Math 65 (2), 1989, p 165-196.

[Gu-Ta] Guivarc'h-Taylor: The Martin compactification of the polydisc at the bottom of the spectrum. Colloq. Math 50/51 (1990), p 537-546.

[Ka] Karpelevitch : The geometry of geodesics and the eigenfunctions of the Laplace Beltrami operator on symmetric spaces. Trans. Moscow Math. Soc. AMS 1967, p 51-199.

[Ko] Koranyi : Poisson integrals and boundary components of symmetric spaces. Invent. Math 434 (1976), p 19-35.

[Mac] Mac Donald : Spherical functions on a group a p-adic type. Univ. of Madras India, 1971.

[Mall] M.P. Malliavin et P. Malliavin : Factorisations et limites de la diffusion horizontale au dessus d'un espace Riemannien symétrique. Théorie du potentiel et analyse harmonique. Springer Lecture Notes in math 404, 1974.

[Mar] Margulis : Discrete subgroups of semi-simple Lie groups. Ergebnisse Math Springer, 1989.

[Moo1] Moore : Compactifications of symmetric spaces I-II. Amer. J. Math. 86 (1964), p 201-218 et 358-378.

[Mo02] Moore : Amenable subgroups of semi-simple groups and proximal flows. Israël J. Math. 34 (1979), p 121-138.

[No] Nolde: Non negative eigenfunctions of Laplace-Beltrami operator on complex symmetric spaces. Uspekhi Math. Nank 21, $n^{\circ} 5,1966, \mathrm{p} 260-261$.

[011] Olshanetsky : Martin boundary for the Laplace-Beltrami operator on a Riemannian space of non positive curvature. Usp. Math. Nauk $24, \mathrm{n}^{\circ} 6$ (1969), p 189-190.

[012] Olshanetsky : Martin boundaries for real semi-simple Lie groups. Journal of Functional Analysis, vol. 126, $\mathrm{n}^{\circ} 1,1994, \mathrm{p}$ 167-216.

[Pi-Wo] The full Martin boundary of a bitree. Preprint Milano, 1992.

[Sa] Satake :On representations and compactifications of symmetric spaces. Annals of Math. vol. $71 \mathrm{n}^{\circ} 1,1960, \mathrm{p} 77-110$.

[Zi] Zimmer : Ergodic theory and semi-simple groups. Birkhaüser, 1984. 